\title{
Decomposition driven interface evolution for layers of binary mixtures. I. Model derivation and stratified base states
}

\author{
Uwe Thiele ${ }^{a)}$ \\ Institut für Physik, Universität Augsburg, D-86135 Augsburg, Germany \\ and Max-Planck-Institut für Physik komplexer Systeme, Nöthnitzer Str. 38, D-01187 Dresden, Germany \\ Santiago Madruga ${ }^{\text {b) }}$ \\ Max-Planck-Institut für Physik komplexer Systeme, Nöthnitzer Str. 38, D-01187 Dresden, Germany \\ Lubor Frastia ${ }^{\mathrm{c})}$ \\ Department of Chemical Engineering, Technion-Israel Institute of Technology, Haifa 32000, Israel
}

(Received 20 July 2007; accepted 15 November 2007; published online 26 December 2007)

\begin{abstract}
A dynamical model is proposed to describe the coupled decomposition and profile evolution of a free surface film of a binary mixture. An example is a thin film of a polymer blend on a solid substrate undergoing simultaneous phase separation and dewetting. The model is based on model-H describing the coupled transport of the mass of one component (convective Cahn-Hilliard equation) and momentum (Navier-Stokes-Korteweg equations) supplemented by appropriate boundary conditions at the solid substrate and the free surface. General transport equations are derived using phenomenological nonequilibrium thermodynamics for a general nonisothermal setting taking into account Soret and Dufour effects and interfacial viscosity for the internal diffuse interface between the two components. Focusing on an isothermal setting the resulting model is compared to literature results and its base states corresponding to homogeneous or vertically stratified flat layers are analyzed. () 2007 American Institute of Physics. [DOI: 10.1063/1.2824404]
\end{abstract}

\section{INTRODUCTION}

Driven by applications in coating technology, micro- and nanostructuring of soft matter layers, and, in general, the development of (multi-)functional surfaces the understanding of thin films of simple and complex fluids is of growing importance. Recent years have seen on the one hand major advances in experimental techniques of preparation and analysis and on the other hand intense developments of the theoretical description of the statics and dynamics of homogeneous and structured films. ${ }^{1-9}$ The dynamics of the structuring often represents examples for micro- and nanofluidic flows, a present focus of interest in its own right. ${ }^{10,11}$

For thin one-layer free surface films of one-component simple or polymeric liquids experimental results, ${ }^{8,12-14}$ and theoretical understanding ${ }^{15-20,14}$ are well developed. However, experiments increasingly focus on complex situations like the evolution of multilayer films of partially miscible ${ }^{21}$ or immiscible ${ }^{4}$ liquids, complex fluids like polymer blends that might undergo dewetting or/and decomposition ${ }^{21-23}$ or solutions of polymers, nanoparticles, colloids or polymer blends with interacting convective motion, phase separation, evaporation/condensation, and evolving rheology. ${ }^{24-39}$ Theoretical descriptions exist, however, only for a small part of the experimentally known complex scenario and phenomena involving free surface thin films. Recent advances include a

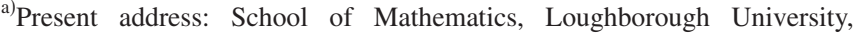
Loughborough, Leicestershire, LE11 3TU, United Kingdom. URL: http:// www.uwethiele.de. Electronic mail: thiele@pks.mpg.de.

${ }^{\text {b) }}$ Electronic mail: santiago@pks.mpg.de, smadruga@gmail.com.

c)Electronic mail: 1frastia@gmail.com.
}

fully nonlinear thin film description in long wave approximation for two layers of immiscible liquids under air ${ }^{40-44}$ and between two plates, ${ }^{45}$ the analysis of the dewetting behavior on chemically or topographically heterogeneous substrates ${ }^{46-49}$ the study of the dynamics of depinning of a driven drop on a heterogeneous substrate,$^{50}$ the description of films with surface active nanoparticles, ${ }^{51}$ and the inclusion of evaporation/condensation in the thin film description. ${ }^{19,51-56}$

Thin films of polymer blends are one of the "simplest" complex systems listed above and extensive experimental results can be found in the literature. ${ }^{4,21,22,57-67}$ Described effects include the dependency of the evolving structure (stratified bi- or trilayer structure that is laterally homogeneous; purely lateral phase separation, checkerboard structure) on substrate properties, ${ }^{57,62,68,69}$ surface roughening or film morphology changes during phase separation, ${ }^{63,66,67,70}$ surface directed spinodal decomposition, ${ }^{68}$ subsequent vertical phase separation and dewetting, ${ }^{21,22}$ and surface phase inversion. ${ }^{59}$ The influence of heterogeneous substrates was also studied. ${ }^{64,71}$ However, as detailed below, to our knowledge there exists no theoretical description of the involved processes that takes into account the evolving composition of the mixture and the evolving surface profile of the film.

The aim of the present paper is to present such a description based on the underlying transport equations. To cover the coupled time evolution of the film thickness and concentration profiles one has to supplement the coupled transport equations for momentum and concentration by appropriate boundary conditions at the free surface and at the solid substrate. 
Two groups of studies are present in the literature that address part of the involved questions. On the one hand, the classical Cahn-Hilliard model ${ }^{72}$ describing purely diffusive decomposition of a binary mixture was studied for films in a gap between two solid plates. ${ }^{7-75}$ At the plates boundary conditions prescribe zero diffusive flow through the plates, energetic preference of the plates for one component and an enforced (or reduced) demixing at the plates. However, such a model can in principle not account for an evolving surface deflection in decomposing films as observed in phaseseparating polymer blends on homogeneous ${ }^{63,66,67,70}$ or patterned $^{64,71}$ substrates. An evolving film profile is by definition related to a convective flow of the mixture.

On the other hand, the coupling of momentum and concentration transport for weakly miscible fluids (i.e., decomposing mixtures) is well studied for bulk systems using the so-called model-H. ${ }^{76-81}$ It couples the convective CahnHilliard equation and the Navier-Stokes equations amended by a concentration dependent stress tensor, the so-called Korteweg stresses. ${ }^{82}$ For a survey of the history of the model, see Ref. 83. A variant of model-H is also used for the dynamics of momentum and density of a single component fluid near a liquid-gas phase transition in isothermal ${ }^{77,84-86}$ or nonisothermal conditions. ${ }^{78,87,88}$ Two-phase liquids and binary mixtures between two solid plates are also investigated. ${ }^{88-90}$ A similar model for miscible liquids was studied, for instance, in Refs. 83 and 91. There the convective Cahn-Hilliard equation is replaced by a "normal" convective diffusion equation. Nowadays, model-H is also applied to multiphase flows in closed microchannels of different geometries (straight quadratic channel, ${ }^{92}$ T-junction ${ }^{93}$ ).

To describe a film of a mixture under air, model- $\mathrm{H}$ is used to describe the dynamics within the film, i.e., modeling the creation and evolution of "internal" diffuse interfaces within the film. The bulk model has to be completed by boundary conditions at the solid substrate (discussed in Refs. 94-96) and by boundary conditions at the free surface. The latter represents an "external" sharp interface. The interaction of the internal diffuse interfaces with the external sharp one via a solutal Marangoni effect results in an additional driving force of the evolution.

Other related work involves an ad hoc lubrication approximation model coupling evolution equations for the film thickness and the mean concentration in the film. ${ }^{97,98} \mathrm{We}$ believe that such a model might be correct in the limit of weak vertical variation of concentration but is not able to describe vertically stratified films and their evolution. An alternative approach uses microscopic discrete models like lattice gas (for results on a binary alloy, see Refs. 99 and 100) or molecular dynamics. ${ }^{101}$ This leads, however, often to a strong restriction in length and time scales that can be studied.

We present our work as a sequence of papers. The first part derives and discusses the basic transport equations and analyzes steady base states. The second part will perform a detailed stability analysis with respect to transversal instability modes for the various qualitatively different base states. Thereby the consequences of convective transport are stud- ied in detail and the sequence of patterning processes is predicted. A planned further sequel will focus on the nonlinear evolution.

The present paper is structured as follows: In Sec. II we derive the coupled transport equations for momentum, density, and temperature in the framework of phenomenological nonequilibrium thermodynamics. After discussing the physical interpretation of the individual contributions representing, for instance, an internal Soret effect (i.e., Soret effect for the internal diffuse interface) and internal interface viscosity, the model is simplified assuming an isothermal setting, vanishing interface viscosity, and internal energies resulting from a setting close to the critical point of demixing. The resulting model-H is compared to versions found in the literature discussing the issue of defining pressure and chemical potential. Section III introduces boundary conditions at the rigid solid substrate and the free surface. It is explained in detail why the incorporation of convective flow is a necessary precondition to describe evolving surface deflections. Next, Secs. IV and V introduce the nondimensionalization and local energies, respectively. Homogeneous and vertically stratified, transversally homogeneous steady state solutions are analyzed in Sec. VI. Section VII summarizes, compares to the literature, and gives an outlook on the sequel. The Appendix uses variational calculus to independently derive the boundary conditions in the static limiting case.

\section{DERIVATION OF EXTENDED MODEL-H}

First we present a derivation of an extended model- $\mathrm{H}$ that accounts for all cross couplings of the transport equations for momentum, concentration, and temperature. This includes Soret and Dufour effects with nonlinear coefficients and interface viscosity for the diffuse interface. It follows in spirit the derivations of the Navier-Stokes equations given in Refs. 102 and 103.

\section{A. General transport equations \\ 1. Conserved quantities}

The starting point consists of the transport, conservation, and balance laws for the relevant phenomenological thermodynamic entities. In general, we have for a conserved scalar or vector field $a(\mathbf{x}, t)$ the transport equation

$$
\frac{\partial}{\partial t} a+\nabla \cdot \mathbf{j}_{a}^{\prime}=0,
$$

where $\mathbf{j}_{a}^{\prime}$ is a general flux density that is a vector or second order tensor. Note that a dotless product always corresponds to a tensor (or outer) product, whereas a dot "." product is an inner product (resulting in a tensor of the order $n-2$, where $n$ is the order of the respective tensor product). The contribution by convective transport with the velocity $\mathbf{v}$ is expressed explicitly by $\mathbf{j}_{a}^{\prime}=\mathbf{j}_{a}+a \mathbf{v}$, where $\mathbf{j}_{a}$ is the diffusive flux caused by (several) microscopic mechanisms.

By definition the total mass density $\rho(\mathbf{x}, t)$ is transported by convection only, i.e., the mass density flux is 


$$
\mathbf{j}_{\rho}^{\prime} \equiv \rho \mathbf{v}=\mathbf{g}
$$

corresponding to the momentum density g. The used convention for the velocity $\mathbf{v}$ is discussed at the end of the present section.

The transport equation for the density (continuity equation) is

$$
\frac{\partial}{\partial t} \rho+\nabla \cdot(\rho \mathbf{v})=0
$$

The density of the momentum $\mathbf{g}$ as well as the density of the total energy $\epsilon$ are transported by convective and diffusive fluxes, i.e.,

$$
\begin{aligned}
& \frac{\partial}{\partial t} \mathbf{g}+\nabla \cdot \underline{\mathbf{j}}_{\mathbf{g}}^{\prime}=0, \\
& \frac{\partial}{\partial t} \boldsymbol{\epsilon}+\nabla \cdot \mathbf{j}_{\epsilon}^{\prime}=0,
\end{aligned}
$$

where $\mathbf{j}_{\mathbf{g}}^{\prime}$ is the tensor of the momentum flux density and $\mathbf{j}_{\epsilon}^{\prime}$ is the energy flux density. Note that all our densities are per volume. Special care has to be taken when comparing with approaches where all or part of the densities are defined as "specific densities" denoting, e.g., in Refs. 89 and 81 densities per unit mass.

By explicitly denoting the transport by convection as before, $\mathbf{j}_{\epsilon}^{\prime}=\boldsymbol{\epsilon}+\mathbf{j}_{\epsilon}$ and $\underline{\mathbf{j}}_{\mathbf{g}}^{\prime}=\mathbf{v g}+\underline{\boldsymbol{\sigma}}$, where $\underline{\boldsymbol{\sigma}}$ is the usual symbol for the diffusive momentum flux $\underline{\mathbf{j}}_{\mathbf{g}}$ (sometimes also called pressure tensor corresponding to the negative of the stress tensor). We will use underlined symbols to denote tensors of second or higher order. Equations (5) and (4) result in

$$
\frac{\partial}{\partial t} \epsilon+\nabla \cdot(\epsilon \mathbf{v})+\nabla \cdot \mathbf{j}_{\epsilon}=0
$$

and

$$
\frac{\partial}{\partial t} \mathbf{g}+\nabla \cdot(\mathbf{v g})+\nabla \cdot \underline{\boldsymbol{\sigma}}=0
$$

respectively.

Introducing the material time derivative $D / D t=\partial / \partial t$ $+\mathbf{v} \cdot \nabla$ one obtains for the velocity field [Eqs. (7) and (3)]

$$
\rho \frac{D \mathbf{v}}{D t}+\nabla \cdot \underline{\boldsymbol{\sigma}}=0
$$

For a binary mixture of fluids a transport equation for the mass density of one of the components has to be added beside the one for the total density $\rho$. Choosing $\rho_{1}$ we have

$$
\frac{\partial}{\partial t} \rho_{1}+\nabla \cdot \mathbf{j}_{\rho_{1}}^{\prime}=0
$$

i.e.,

$$
\frac{\partial}{\partial t} \rho_{1}+\nabla \cdot\left(\rho_{1} \mathbf{v}\right)+\nabla \cdot \mathbf{j}_{\rho_{1}}=0
$$

The density of the other component is $\rho_{2}=\rho-\rho_{1}$. Its transport is described by a similar equation as Eq. (10). The requirement that adding the equations for $\rho_{1}$ and $\rho_{2}$ gives Eq. (3) determines $\mathbf{j}_{\rho_{1}}=-\mathbf{j}_{\rho_{2}}$ (cf. Ref. 89) as can also be seen from the general form of $\mathbf{j}_{\rho_{1}}$ derived later in Eq. (33). As discussed in detail in Ref. 104 the latter condition defines the velocity $\mathbf{v}$ used in the transport equations for the mass densities as the mass-averaged velocity, i.e., the velocity also used in the momentum equation.

We have now discussed all the conserved quantities. The conservation of angular momentum is guaranteed by the symmetry properties of the stress tensor (see below). ${ }^{102} \mathrm{Next}$ we discuss the transport equations for nonconserved quantities.

\section{Nonconserved quantities}

The transport equations for nonconserved quantities contain additional source terms, i.e., for a general field $a$ one writes

$$
\frac{\partial a}{\partial t}+\nabla \cdot \mathbf{j}_{a}^{\prime}=Q_{a}
$$

where $Q_{a}$ is a possibly space- and time-dependent source density. Relevant nonconserved quantities are the densities of the internal energy $u$ and of the entropy $s$.

For systems with small gradients of concentration and/or temperature the energies do only depend on the local fields. For strong gradients, however, this statement does not hold any more and the energy of a system will depend also on field gradients. In the present case we consider strong density gradients related to diffuse interfaces between different phases. The underlying assumption is that for a demixing system gradients in $\rho_{1}$ might be much larger than all other gradients. The latter enter the theory as parametric dependencies on space only.

We define the internal energy $u\left(\rho, \rho_{1}, s, t\right)$ as the thermodynamic equilibrium value for a local fluid element, i.e., it shall not depend on gradients. The conserved total energy $\epsilon$, however, shall include gradient terms in $\rho_{1}$. The relation between the two is

$$
\epsilon=u+\frac{\rho}{2} v^{2}+\frac{\xi}{2}\left(\nabla \rho_{1}\right)^{2}
$$

Note that the unit of $\xi$ is $[\xi]=\mathrm{m}^{7} /\left(\mathrm{kg} \mathrm{s}^{2}\right)$. The energy densities have units $[\epsilon]=[u]=\mathrm{N} \mathrm{m} / \mathrm{m}^{3}=\mathrm{kg} /\left(\mathrm{m} \mathrm{s}^{2}\right)$. The transport equation for the internal energy is

$$
\frac{\partial u}{\partial t}+\nabla \cdot \mathbf{j}_{u}^{\prime}=Q_{u}
$$

with

$$
\mathbf{j}_{u}^{\prime}=u \mathbf{v}+\mathbf{j}_{u}
$$

For the irreversible processes in question entropy is not conserved. The transport equation for its volume density is 


$$
\frac{\partial s}{\partial t}+\nabla \cdot \mathbf{j}_{s}^{\prime}=Q_{s}=\frac{R}{T}
$$

with $\mathbf{j}_{s}^{\prime}=s \mathbf{v}+\mathbf{j}_{s}$ the total entropy flux density. We write the source density $Q_{s}$ in the usual form $R / T$ where $R$ is the so-called dissipation function and $T$ is the temperature. ${ }^{102}$

\section{B. Determination of thermodynamic forces}

The flux densities $\underline{\boldsymbol{\sigma}}, \mathbf{j}_{\epsilon}, \mathbf{j}_{u}, \mathbf{j}_{\rho_{1}}, \mathbf{j}_{s}$, and source terms $R / T$, $Q_{u}$ remain to be determined. The specific transport equation for the internal energy is obtained from the transport equation of the total energy Eq. (6) using Eqs. (3), (7), (10), and (12). It reads

$$
\begin{aligned}
\frac{D}{D t} u & +u \nabla \cdot \mathbf{v}+\nabla \cdot \mathbf{j}_{u} \\
= & \left\{-\underline{\boldsymbol{\sigma}}+\xi\left(\nabla \rho_{1}\right)\left(\nabla \rho_{1}\right)\right. \\
& \left.-\xi\left[\frac{1}{2}\left(\nabla \rho_{1}\right)^{2}+\rho_{1} \Delta \rho_{1}\right] \underline{\mathbf{I}}\right\}: \nabla \mathbf{v}-\xi\left(\Delta \rho_{1}\right) \nabla \cdot \mathbf{j}_{\rho_{1}}
\end{aligned}
$$

with

$$
\mathbf{j}_{u}=\mathbf{j}_{\epsilon}-\mathbf{v} \cdot \underline{\boldsymbol{\sigma}}-\xi\left(\nabla \rho_{1}\right)\left[\rho_{1}(\nabla \cdot \mathbf{v})+\nabla \cdot \mathbf{j}_{\rho_{1}}\right] .
$$

The symbol ":" stands for a double inner product, i.e., $\underline{\mathbf{a}}: \underline{\mathbf{b}}=\sum_{i j} a_{i j} b_{j i}$.

The time evolution of the entropy is deduced using a local form of Gibbs relation for each fluid element, i.e., from the assumption that small fluid elements are in thermodynamic equilibrium. Gibbs relation for a local fluid element of volume $V$ writes

$$
d U=T d S-p d V+\hat{\mu}_{1} d N_{1}+\hat{\mu}_{2} d N_{2},
$$

where $U, S, p, \hat{\mu}_{1}, \hat{\mu}_{2}, N_{1}, N_{2}$ stand for internal energy, entropy, pressure, chemical potentials of component 1 and 2 , and particle numbers of component 1 and 2 . The chemical potentials (with hat) are related to particle numbers. The relation (18) is transformed expressing extensive variables by the corresponding densities using $U=u V, \quad N_{i}=n_{i} V$ $=\rho_{i} N_{a} / M_{i} V, N=N_{1}+N_{2}, \rho_{2}=\rho-\rho_{1}$, and $S=s V$, where $N$ is the total particle number, $M_{i}$ is the molar mass of component $i$, and $N_{a}$ is the Avogadro number. One obtains

$$
\begin{aligned}
d u= & T d s+\mu_{2} d \rho+\mu_{d} d \rho_{1} \\
& +\left(-u+\mu_{2} \rho+\mu_{d} \rho_{1}+T s-p\right) d V / V .
\end{aligned}
$$

The chemical potential $\mu_{2}=\hat{\mu}_{2} N_{a} / M_{2}$ of component 2 and the difference of the chemical potentials of components 1 and $2 \mu_{d}=\hat{\mu}_{1} N_{a} / M_{1}-\hat{\mu}_{2} N_{a} / M_{2}$ are related to volume densities and have units $\left[\mu_{2}\right]=\left[\mu_{d}\right]=\mathrm{m}^{2} / \mathrm{s}^{2}$.

Relation (19) is valid for arbitrary local volume $V$, i.e., one obtains the local Gibbs relation

$$
d u=T d s+\mu_{2} d \rho+\mu_{d} d \rho_{1}
$$

and the local Gibbs-Duhem relation

$$
p=-u+T s+\mu_{2} \rho+\mu_{d} \rho_{1} .
$$

Here, we observe that, within the framework of volume density quantities, $p$ behaves as a thermodynamic potential that is related to $u$ by the Legendre transform (21). Furthermore,

$$
T=\left(\frac{\partial u}{\partial s}\right)_{\rho, \rho_{1}}, \quad \mu_{2}=\left(\frac{\partial u}{\partial \rho}\right)_{s, \rho_{1}}, \quad \text { and } \mu_{d}=\left(\frac{\partial u}{\partial \rho_{1}}\right)_{s, \rho} .
$$

Equation (20) is divided by a small time span $d t$ that is, however, large as compared to typical microscopic time scales yielding

$$
\frac{d u}{d t}=T \frac{d s}{d t}+\mu_{2} \frac{d \rho}{d t}+\mu_{d} \frac{d \rho_{1}}{d t} .
$$

This relation is valid in all local volume elements that might be convected by the flow, i.e., the derivatives $d / d t$ correspond to Lagrangian or material time derivatives denoted above $D / D t$. Using Eqs. (3), (10), and (16) one transforms Eq. (23) into the wanted form of Eq. (15),

$$
\begin{aligned}
\frac{\partial s}{\partial t}+\nabla \cdot & {\left[s \mathbf{v}+\frac{\mathbf{j}_{u}}{T}+\frac{\mathbf{j}_{\rho_{1}}}{T}\left(\xi \Delta \rho_{1}-\mu_{d}\right)\right] } \\
= & \frac{1}{T}\left\{-\boldsymbol{\sigma}+\xi\left(\nabla \rho_{1}\right)\left(\nabla \rho_{1}\right)\right. \\
& \left.+\left[p-\xi \rho_{1} \Delta \rho_{1}-\frac{\xi}{2}\left(\nabla \rho_{1}\right)^{2}\right] \underline{\mathbf{I}}\right\}:(\nabla \mathbf{v})+\mathbf{j}_{u} \cdot \nabla\left(\frac{1}{T}\right) \\
& +\mathbf{j}_{\rho_{1}} \cdot \nabla\left(\frac{\xi \Delta \rho_{1}-\mu_{d}}{T}\right)
\end{aligned}
$$

with the pressure $p$ and the flux of internal energy $\mathbf{j}_{u}$ given by Eqs. (21) and (17), respectively. Here it is already possible to see the structure of the dissipative contribution to the pressure or stress tensor (the part in \{\} on the rhs). The reversible entropy transport [cf. Eqs. (15) and (24)], i.e., the entropy flux

$$
\mathbf{j}_{s}^{\prime}=s \mathbf{v}+\frac{\mathbf{j}_{u}}{T}+\frac{\mathbf{j}_{\rho_{1}}}{T}\left(\xi \Delta \rho_{1}-\mu_{d}\right)
$$

contains the convective transport, the transport via the heat flux, and the transport via the diffusive flux of species one. Comparing Eqs. (15) and (24) allows us to identify the source term for the entropy. It is related to irreversible processes and written in terms of the dissipation function

$$
\begin{aligned}
R= & \left\{-\underline{\boldsymbol{\sigma}}+\xi\left(\nabla \rho_{1}\right)\left(\nabla \rho_{1}\right)\right. \\
& \left.+\left[p-\xi \rho_{1} \Delta \rho_{1}-\frac{\xi}{2}\left(\nabla \rho_{1}\right)^{2}\right] \underline{\mathbf{I}}\right\}:(\nabla \mathbf{v})+T \mathbf{j}_{u} \cdot \nabla\left(\frac{1}{T}\right) \\
& +T \mathbf{j}_{\rho_{1}} \cdot \nabla\left(\frac{\xi \Delta \rho_{1}-\mu_{d}}{T}\right) .
\end{aligned}
$$

We can directly deduce the reversible part $\underline{\boldsymbol{\sigma}}^{r}$ of the pressure tensor $\underline{\boldsymbol{\sigma}}=\underline{\boldsymbol{\sigma}}^{r}+\underline{\boldsymbol{\sigma}}^{d}$, because only the dissipative part $\underline{\boldsymbol{\sigma}}^{d}$ contributes to the dissipation function, i.e., 


$$
\underline{\boldsymbol{\sigma}}^{r}=\xi\left(\nabla \rho_{1}\right)\left(\nabla \rho_{1}\right)+\left[p-\xi \rho_{1} \Delta \rho_{1}-\frac{\xi}{2}\left(\nabla \rho_{1}\right)^{2}\right] \underline{\mathbf{I}} .
$$

Note that the negative of $\underline{\boldsymbol{\sigma}}^{r}-p \underline{\mathbf{I}}$ is known as the capillary or Korteweg stress tensor in the literature. ${ }^{77,78,82,84}$ The dissipative part $\underline{\boldsymbol{\sigma}}^{d}$ is also called viscose pressure tensor or friction tensor. The dissipation function has the structure $R=\Sigma_{\alpha \alpha} \mathbf{j}_{\alpha} \cdot \mathbf{f}_{\alpha}$, where the $\mathbf{j}_{\alpha}$ and $\mathbf{f}_{\alpha}$ are general thermodynamic fluxes and forces, respectively, that might be tensors. Correspondingly the "." stands here for a "complete" inner product (scalar product).

We have the fluxes $\mathbf{j}_{u}, \mathbf{j}_{\rho_{1}}$, and $-\underline{\boldsymbol{\sigma}}^{d}$ with the corresponding forces

$$
\begin{aligned}
& \mathbf{f}_{u}=T \nabla\left(\frac{1}{T}\right), \\
& \mathbf{f}_{\rho_{1}}=T \nabla\left(\frac{\xi \Delta \rho_{1}-\mu_{d}}{T}\right), \\
& \mathbf{f}_{\mathbf{g}}=\nabla \mathbf{v} .
\end{aligned}
$$

In the last step of the derivation, the thermodynamic fluxes have to be determined. Following Onsager, we first make the basic ansatz of linear nonequilibrium thermodynamics, i.e., we postulate a linear dependence of the fluxes on all the forces (if symmetry permits), i.e.,

$$
\mathbf{j}_{\beta}=\sum_{\alpha} \underline{\mathbf{L}}_{\beta \alpha} \cdot \mathbf{f}_{\alpha}
$$

with $\underline{\mathbf{L}}_{\alpha \beta}=\underline{\mathbf{L}}_{\beta \alpha}$ (Onsager relation, resulting from microscopic reversibility). Specifically, we get for the fluxes

$$
\begin{aligned}
& \mathbf{j}_{u}=T \underline{\mathbf{L}}_{u u} \cdot \nabla\left(\frac{1}{T}\right)+T \underline{\mathbf{L}}_{u \rho_{1}} \cdot \nabla\left(\frac{\xi \Delta \rho_{1}-\mu_{d}}{T}\right), \\
& \mathbf{j}_{\rho_{1}}=T \underline{\mathbf{L}}_{\rho_{1} u} \cdot \nabla\left(\frac{1}{T}\right)+T \underline{\mathbf{L}}_{\rho_{1} \rho_{1}} \cdot \nabla\left(\frac{\xi \Delta \rho_{1}-\mu_{d}}{T}\right), \\
& \underline{\boldsymbol{\sigma}}^{d}=-\underline{\mathbf{L}}_{\mathbf{g g}}: \nabla \mathbf{v} .
\end{aligned}
$$

The cross-coupling terms in $\mathbf{j}_{u}$ and $\mathbf{j}_{\rho_{1}}$ correspond to (generalized) Dufour and Soret effects, respectively. Note that there is no linear coupling between the momentum flux and the thermodynamic forces corresponding to temperature and concentration gradients. However, when discussing the total energy for systems with large gradients in the density $\rho_{1}$ we included quadratic terms in the density gradient. For consistency, a nonlinear term, quadratic in the forces $\mathbf{f}_{\rho_{1}}$, should be added to relation (34) resulting in

$$
\underline{\boldsymbol{\sigma}}^{d}=-\underline{\mathbf{L}}_{\mathbf{g g}}: \nabla \mathbf{v}-T^{2} \underline{\mathbf{Q}}_{\mathbf{g} \rho_{1}}:\left(\nabla \frac{\xi \Delta \rho_{1}-\mu_{d}}{T}\right)\left(\nabla \frac{\xi \Delta \rho_{1}-\mu_{d}}{T}\right) .
$$

The additional term is related to irreversible aspects of the dynamics of the diffuse interface and can be seen as a generalization of the term $\underline{\mathbf{S}}_{*}$ proposed in the conclusion of Ref. 89 that reads

$$
\underline{\mathbf{S}}_{*}=\beta(\nabla \zeta)(\nabla \zeta),
$$

where $\beta$ is an undetermined empirical coefficient and $\zeta$ is the difference between the chemical potentials of components 1 and 2 for an inhomogeneous equilibrium which in our terms is $\zeta=\mu_{d}-\xi \Delta \rho_{1}$ as is discussed below, after Eq. (57) and also in Ref. 77. $\underline{\mathbf{Q}}_{\mathbf{g}_{1}}$ corresponds to a tensor of interfacial viscosities. Related issues are discussed for sharp interface theories in Ref. 105.

The $\underline{\mathbf{L}}_{\alpha \beta}$ are tensors of various orders: $\underline{\mathbf{L}}_{u u}$ is of order 2, whereas $\underline{\mathbf{L}}_{\mathbf{g g}}$ and $\underline{\mathbf{Q}}_{\mathbf{g} \rho_{1}}$ are of order 4 (i.e., in the general case there are $3^{4}=81$ components: viscosities). Assumption of an isotropic medium significantly reduces the number of coefficients. $^{102}$

Considering small interfacial viscosities only, we neglect the corresponding terms and finally get

$$
\begin{aligned}
& \mathbf{j}_{u}=-\frac{k_{1}}{T} \nabla T+k_{2} T \nabla\left(\frac{\xi \Delta \rho_{1}-\mu_{d}}{T}\right), \\
& \mathbf{j}_{\rho_{1}}=-\frac{k_{2}}{T} \nabla T+k_{3} T \nabla\left(\frac{\xi \Delta \rho_{1}-\mu_{d}}{T}\right), \\
& \underline{\boldsymbol{\sigma}}^{d}=-\zeta \underline{\mathbf{I}}(\nabla \cdot \mathbf{v})-\eta\left[\nabla \mathbf{v}+(\nabla \mathbf{v})^{T}-\frac{2}{3} \underline{\mathbf{I}}(\nabla \cdot \mathbf{v})\right],
\end{aligned}
$$

where $\zeta$ and $\eta$ are the dynamic and shear bulk viscosity, respectively, and the kinetic coefficients have the units $\left[k_{1}\right]=\mathrm{kg} \mathrm{m} / \mathrm{s}^{3}, \quad[\eta]=[\zeta]=\left[k_{2}\right]=\mathrm{kg} / \mathrm{m} \mathrm{s}$ and $\left[k_{3}\right]=\mathrm{kg} \mathrm{s} / \mathrm{m}^{3}$. The governing equations are now obtained by introducing the fluxes Eqs. (37)-(39) into the corresponding transport equations. Introducing Eq. (38) into Eq. (10) we get for the transport of $\rho_{1}$,

$$
\begin{aligned}
& \frac{\partial}{\partial t} \rho_{1}+\nabla \cdot\left(\rho_{1} \mathbf{v}\right)+\nabla \cdot\left[-\frac{k_{2}}{T} \nabla T+k_{3} T \nabla\left(\frac{\xi \Delta \rho_{1}-\mu_{d}}{T}\right)\right] \\
& \quad=0
\end{aligned}
$$

and for momentum transport feeding Eqs. (39) and (27) into Eq. (8) we have

$$
\begin{aligned}
\rho \frac{\partial \mathbf{v}}{\partial t}+ & \rho \mathbf{v} \cdot \nabla \mathbf{v} \\
= & -\nabla \cdot\left\{\xi\left(\nabla \rho_{1}\right)\left(\nabla \rho_{1}\right)+\left[p-\xi \rho_{1} \Delta \rho_{1}-\frac{\xi}{2}\left(\nabla \rho_{1}\right)^{2}\right] \underline{\mathbf{I}}\right. \\
& \left.-\zeta \underline{\underline{I}}(\nabla \cdot \mathbf{v})-\eta\left[\nabla \mathbf{v}+(\nabla \mathbf{v})^{T}-\frac{2}{3} \underline{\mathbf{I}}(\nabla \cdot \mathbf{v})\right]\right\} .
\end{aligned}
$$

Assuming that $\mu_{2}$ and $\mu_{d}$ are given, to obtain a closed set of equations we still need an equation for the evolution of the temperature field. We obtain it by multiplying the transport equation for entropy Eq. (24) with $T$, and expressing the entropy via the Gibbs relation in a similar way as Batchelor (pp. 35ff and 136ff of Ref. 102).

After reordering Eq. (24) yields 


$$
T \frac{D s}{D t}+T s \nabla \cdot \mathbf{v}=-T \nabla \cdot\left[\frac{\mathbf{j}_{u}}{T}+\frac{\mathbf{j}_{\rho_{1}}}{T}\left(\xi \Delta \rho_{1}-\mu_{d}\right)\right]+R .
$$

All terms on the rhs are already known. We next determine the lhs terms. Consistent with Gibbs relation (18) one can express the entropy as a function of $T, V, N_{1}$ and $N_{2}$. Using the second law of thermodynamics we write

$$
\begin{aligned}
d Q=T d S= & C_{V} d T+T\left(\frac{\partial S}{\partial V}\right)_{T, N_{i}} d V+T\left(\frac{\partial S}{\partial N_{1}}\right)_{T, V, N_{2}} d N_{1} \\
& +T\left(\frac{\partial S}{\partial N_{2}}\right)_{T, V, N_{1}} d N_{2}
\end{aligned}
$$

where $Q$ stands for the heat supplied to the system. Fixing $N_{1}$ and $N_{2}$ the last two terms vanish. Expressing volume $V$ in terms of $T, p, N_{1}$, and $N_{2}$, assuming fixed $N_{1}$ and $N_{2}$ yields

$$
d V=\left(\frac{\partial V}{\partial T}\right)_{p, N_{i}} d T+\left(\frac{\partial V}{\partial p}\right)_{T, N_{i}} d p .
$$

Substituting into Eq. (43) we obtain (assuming fixed $N_{1}$ and $\left.N_{2}\right)$

$$
\begin{aligned}
T d S= & {\left[C_{V}+T\left(\frac{\partial S}{\partial V}\right)_{T, N_{i}}\left(\frac{\partial V}{\partial T}\right)_{p, N_{i}}\right] d T } \\
& +T\left(\frac{\partial S}{\partial V}\right)_{T, N_{i}}\left(\frac{\partial V}{\partial p}\right)_{T, N_{i}} d p=C_{p} d T+T\left(\frac{\partial S}{\partial p}\right)_{T, N_{i}} d p .
\end{aligned}
$$

Introducing the thermal expansion coefficient

$$
\beta=\frac{1}{V}\left(\frac{\partial V}{\partial T}\right)_{p, N, N_{1}}
$$

and comparing the respective prefactors of $d T$ in the two lines of Eq. (44) we rewrite Eq. (43) as

$$
\begin{aligned}
d Q= & T d S=C_{V} d T+\frac{C_{p}-C_{V}}{\beta V} d V+T\left(\frac{\partial S}{\partial N_{1}}\right)_{T, V, N_{2}} d N_{1} \\
& +T\left(\frac{\partial S}{\partial N_{2}}\right)_{T, V, N_{1}} d N_{2} .
\end{aligned}
$$

Assuming that the material constants $C_{p}, C_{V}$ and $\beta$ are known we next focus on the last two terms of Eq. (45). Consistent with Eq. (18), we write for the Helmholtz free energy, $F=U-T S$, of a local fluid element $V$ in thermodynamic equilibrium

$$
d F=d(U-T S)=-S d T-p d V+\hat{\mu}_{1} d N_{1}+\hat{\mu}_{2} d N_{2} .
$$

Partial differentiation of Eq. (46) with respect to $N_{1}$ and $T$ gives

$$
-\left(\frac{\partial S}{\partial N_{1}}\right)_{T, V, N_{2}}=\left(\frac{\partial}{\partial N_{1}}\left(\frac{\partial F}{\partial T}\right)_{V, N_{i}}\right)_{T, V, N_{2}}
$$

and

$$
\left(\frac{\partial \hat{\mu}_{1}}{\partial T}\right)_{V, N_{i}}=\left(\frac{\partial}{\partial T}\left(\frac{\partial F}{\partial N_{1}}\right)_{T, V, N_{2}}\right)_{V, N_{i}},
$$

respectively. Identifying the mixed second derivatives we obtain the so-called Maxwell relation for $S$ and $\hat{\mu}_{1}$,

$$
\left(\frac{\partial S}{\partial N_{1}}\right)_{T, V, N_{2}}=-\left(\frac{\partial \hat{\mu}_{1}}{\partial T}\right)_{V, N_{i}} .
$$

Analogously, we obtain the Maxwell relation for $S$ and $\hat{\mu}_{2}$. Using them we rewrite Eq. (45) as

$$
\begin{aligned}
T d S= & C_{V} d T+\frac{C_{p}-C_{V}}{\beta V} d V-T\left(\frac{\partial \hat{\mu}_{1}}{\partial T}\right)_{V, N_{i}} d N_{1} \\
& -T\left(\frac{\partial \hat{\mu}_{2}}{\partial T}\right)_{V, N_{i}} d N_{2} .
\end{aligned}
$$

Note that there exist other ways to express the heat. The one chosen here is advantageous because the equation explicitly contains $d V$. This allows us to consider the incompressible case by setting $d V=0$ (see Sec. II C). The dependencies of the chemical potentials on temperature will also be given.

Next, let us rewrite local Gibbs and Gibbs-Duhem relations, Eqs. (20) and (21), in terms of the density of the Helmholtz free energy, $f\left(\rho, \rho_{1}, T, t\right)=u-T s$,

$$
d f=-s d T+\mu_{2} d \rho+\mu_{d} d \rho_{1},
$$

$$
p=-f+\mu_{2} \rho+\mu_{d} \rho_{1}
$$

using the above introduced procedure for obtaining local Gibbs (20) and Gibbs-Duhem (21) relations, we derive from Eq. (49), the relations for the volume densities of the extensive quantities

$$
T d s=c_{V} d T-T\left(\frac{\partial \mu_{2}}{\partial T}\right)_{\rho, \rho_{1}} d \rho-T\left(\frac{\partial \mu_{d}}{\partial T}\right)_{\rho, \rho_{1}} d \rho_{1}
$$

and

$$
T s=\frac{c_{p}-c_{V}}{\beta}-T\left(\frac{\partial \mu_{2}}{\partial T}\right)_{\rho, \rho_{1}} \rho-T\left(\frac{\partial \mu_{d}}{\partial T}\right)_{\rho, \rho_{1}} \rho_{1},
$$

respectively. As above, $\mu_{2}=\hat{\mu}_{2} N_{a} / M_{2}$ and $\mu_{d}=\hat{\mu}_{1} N_{a} / M_{1}$ $-\hat{\mu}_{2} N_{a} / M_{2}$ are related to densities not to particle number. Note that we also changed the notation for partial derivative with respect to $T$ regarding the chemical potentials $\mu_{2}, \mu_{d}$ as defined by Eq. (50).

Finally, dividing Eq. (52) by $d t$, identifying $d / d t$ with the material derivative $D / D t$, substituting into Eq. (42), using Eqs. (3) and (10) and reordering we obtain the transport equation for the temperature field 


$$
\begin{gathered}
c_{V} \frac{D T}{D t}+\frac{c_{p}-c_{V}}{\beta} \nabla \cdot \mathbf{v}+T\left(\frac{\partial \mu_{d}}{\partial T}\right)_{\rho, \rho_{1}} \nabla \cdot \mathbf{j}_{\rho_{1}} \\
=-T \nabla \cdot\left[\frac{\mathbf{j}_{u}}{T}+\frac{\mathbf{j}_{\rho_{1}}}{T}\left(\xi \Delta \rho_{1}-\mu_{d}\right)\right]+R .
\end{gathered}
$$

The system consisting of Eqs. (3), (40), (41), and (54) describes a compressible binary mixture in a nonisothermal setting. Comparison of the general case with Ref. 89 turns out to be rather cumbersome because there specific densities and molar fractions are used. The present system is more complete because our thermodynamic fluxes include all possible thermodynamic forces that were determined beforehand. In contrast, Ref. 89 directly assumes Fick's, Fourier's, and Stokes' law. In the isothermal case our system can be compared to the one derived in Ref. 81. Momentum and total mass density equations agree when taking into account different pressure definitions (also see Sec. II C). However, the isothermal version of our Eq. (40) does not agree with their Eq. (3.34c). The difference can be traced back to their generalized Fick's law [their Eq. (3.24b)]. It is based on a generalized chemical potential derived from a specific free energy density, whereas in our view it should have been based on volume density of the free energy.

\section{Model-H: Bulk equations}

Next, we simplify the coupled equations for temperature, momentum, and volume density of component 1 by assuming a fluid with constant density $\rho$ (which implies incompressibility $\nabla \cdot \mathbf{v}=0$ ), in an isothermal setting (constant $T$ ). This can be done without problems when assuming that the mass densities of the two pure components are identical. For the subtle issues arising for densities that are different, including the question of quasi-incompressibility we refer to Refs. 81 and 104, and references therein. Further, we express the density $\rho_{1}$ in terms of a mass concentration $c_{1}=\rho_{1} / \rho$ and obtain from Eq. (40) the convective Cahn-Hilliard equation

$$
\frac{\partial c_{1}}{\partial t}+\mathbf{v} \cdot \nabla c_{1}+\nabla \cdot\left[M_{1} \nabla\left(\sigma_{c_{1}} \Delta c_{1}-\tilde{\mu}_{d}\right)\right]=0,
$$

where we introduced $\tilde{\mu}_{d}=\rho \mu_{d}, \sigma_{c_{1}}=\rho^{2} \xi$, and $M_{1}=k_{3} / \rho^{2}$. Note that $c_{1}$ is dimensionless and $\left[\sigma_{c_{1}}\right]=\mathrm{m} \mathrm{kg} / \mathrm{s}^{2}$, $\left[\tilde{\mu}_{d}\right]=\mathrm{kg} / \mathrm{m} \mathrm{s}^{2}$, and $\left[M_{1}\right]=\mathrm{s} \mathrm{m}^{3} / \mathrm{kg}$. Care has to be taken when comparing to other authors that use volume or mole fraction instead of the mass concentration used here, e.g., Refs. 89 and 104.

The momentum Eq. (41) reduces to

$$
\begin{aligned}
\rho \frac{\partial \mathbf{v}}{\partial t}+\rho \mathbf{v} \cdot \nabla \mathbf{v}= & -\nabla \cdot\left\{\sigma_{c_{1}}\left(\nabla c_{1}\right)\left(\nabla c_{1}\right)\right. \\
& \left.+\left[p-\sigma_{c_{1}} c_{1} \Delta c_{1}-\frac{\sigma_{c_{1}}}{2}\left(\nabla c_{1}\right)^{2}\right] \underline{\mathbf{I}}\right\}+\eta \Delta \mathbf{v},
\end{aligned}
$$

where the mechanical pressure is given by the local GibbsDuhem relation (21), i.e., $p=-u+\mu_{2} \rho+T s+\tilde{\mu}_{d} c_{1}$. We emphasize that $p$ as the mechanical pressure for a homogeneous material in the thermodynamic equilibrium is a locally defined variable that should not depend on any gradient or derivative. However, for simplicity we introduce an effective pressure $p_{\text {eff }}$ that incorporates all terms in the square brackets in Eq. (56). Equations (55) and (56) are normally called model-H. ${ }^{77}$

In the literature model-H is presented in various forms. Especially, the momentum equation is written in different ways. Most differences arise from different definitions of the pressure $p_{\text {eff }}$ introduced in the last paragraph. In the following we indicate how to translate the different formulations and point out "irreducible" differences.

The review by Anderson et al. ${ }^{77}$ gives the transport equation for the momentum in a binary mixture [their Eq. (17b) with Eqs. (16a) and (19)] in our notation

$$
\begin{aligned}
\rho\left[\frac{\partial}{\partial t} \mathbf{v}+\mathbf{v} \cdot \nabla \mathbf{v}\right]= & -\nabla \cdot\left[\left(p_{A}-\frac{\sigma_{c_{1}}}{2}\left(\nabla c_{1}\right)^{2}\right) \underline{\mathbf{I}}\right. \\
& \left.+\sigma_{c_{1}}\left(\nabla c_{1}\right)\left(\nabla c_{1}\right)\right]+\eta \Delta \mathbf{v} .
\end{aligned}
$$

However, their Eq. (20) for their chemical potential $\mu_{c}$ indicates that they do not follow their Eq. (10), but already absorbed additional terms into their $\mu_{c}$. Our formulation coincides with theirs identifying their $p_{A}-\sigma_{c_{1}}\left(\nabla c_{1}\right)^{2} / 2$ and our $p_{\text {eff }_{1}}$. The difference in the formulation arises because Anderson et al. use $\mu_{c}$ in place of our $\tilde{\mu}_{d}-\sigma_{c_{1}} \Delta c_{1}=\bar{\mu}$ in the thermodynamic pressure definition, i.e., their $\mu_{c}=\bar{\mu}$ is the chemical potential for an inhomogeneous equilibrium. Then also their Eq. (21) corresponds to our Eq. (55).

Jasnow and Viñals ${ }^{78}$ present two forms for the momentum equation

$$
\rho\left[\frac{\partial}{\partial t} \mathbf{v}+\mathbf{v} \cdot \nabla \mathbf{v}\right]=-\nabla p_{\mathrm{JV}}+\eta \Delta \mathbf{v}+\bar{\mu} \nabla c_{1}
$$

[their Eq. (2)] and

$$
\rho\left[\frac{\partial}{\partial t} \mathbf{v}+\mathbf{v} \cdot \nabla \mathbf{v}\right]=-\nabla \tilde{p}_{J V}+\eta \Delta \mathbf{v}-c_{1} \nabla \bar{\mu}
$$

[their Eq. (2) with the replacement described in the last paragraph of their Appendix]. They also use $\bar{\mu}$ instead of $\tilde{\mu}_{d}$. We introduce different symbols $p_{J V}$ and $\tilde{p}_{J V}$ for the respective pressures. The second form can be obtained from ours taking into account $\nabla \cdot\left[p_{\text {eff }_{1}} \underline{\mathbf{I}}+\sigma_{c_{1}}\left(\nabla c_{1}\right)\left(\nabla c_{1}\right)\right]=c_{1} \nabla \bar{\mu}+\nabla p$. The first form just follows from integration by parts and redefining the pressure again: $\tilde{p}_{J V}=p_{J V}-c_{1} \bar{\mu}$.

The form of model-H presented in the review by Hohenberg and Halperin ${ }^{76}$ gives a momentum equation [their Eq. (5.1b)] that agrees on the first view with the second form of Jasnow and Viñals Eq. (59). However, they dropped the pressure term, i.e., in the limit of constant concentration their model does not reduce to the Navier-Stokes equations.

Finally, we rewrite model-H in terms of the difference of concentrations $c=c_{1}-c_{2}=2 c_{1}-1$. Introducing new parameters $\sigma_{c}=\sigma_{c_{1}} / 4$ and $M=4 M_{1}$ and specifying the chemical potential $\tilde{\mu}_{d}=2 \partial_{c} f(c)$, where $f(c)$ is the concentration dependent part of the local free energy, results in 


$$
\partial_{t} c+\mathbf{v} \cdot \nabla c=-\nabla \cdot\left\{M \nabla\left[\sigma_{c} \Delta c-\partial_{c} f(c)\right]\right\}
$$

and

$$
\rho \frac{\partial \mathbf{v}}{\partial t}+\rho \mathbf{v} \cdot \nabla \mathbf{v}=-\nabla \cdot\left\{\sigma_{c}(\nabla c)(\nabla c)+p_{\text {eff }} \mathbf{I}\right\}+\eta \Delta \mathbf{v},
$$

where

$$
p_{\text {eff }}=p-\sigma_{c}(c+1) \Delta c-\frac{\sigma_{c}}{2}(\nabla c)^{2} .
$$

Fixing $f(c)$ to be a symmetric double-well potential, Eq. (60) corresponds to the convective Cahn-Hilliard equation studied, for instance, in Refs. 106 and 107. The energy will be further discussed in Sec. V. Because of its importance for the boundary conditions (see Sec. III) we also give the stress tensor

$$
\underline{\boldsymbol{\tau}}=-p_{\mathrm{eff}} \underline{\mathbf{I}}-\sigma_{c}(\nabla c)(\nabla c)+\eta\left[\nabla \mathbf{v}+(\nabla \mathbf{v})^{T}\right],
$$

where $\left(p-p_{\text {eff }}\right) \underline{\mathbf{I}}-\sigma_{c}(\nabla c)(\nabla c)$ represents the Korteweg stress. ${ }^{82,83}$ The pressure $p_{\text {eff }}$ can be calculated from the Poisson equation

$$
\Delta p_{\text {eff }}=-\sigma_{c}(\nabla \nabla):[(\nabla c)(\nabla c)]-\rho(\nabla \mathbf{v}):(\nabla \mathbf{v}) .
$$

We emphasize that the Korteweg stress is reversible as discussed above at Eq. (27). Reversibility clearly appears using the variational approach in the Appendix that leads to the bulk force equilibrium [discussed below in Eq. (A28)], i.e., the static limit of Eq. (61). Compare also the variational approach in Ref. 81 and the simplified version in the Appendix of Ref. 108.

In the literature the various formulations of model- $\mathrm{H}$ are mainly used to describe the behavior of bulk flows. ${ }^{77-80}$ Systems confined between rigid plates are considered in some cases $^{78,88,89}$ assuming (i) the diffuse interface is far away from the plates, and (ii) the walls are neutral with respect to the two components. However, the role of energetically biased plates and the evolution of a free surface of the binary mixture have to be understood in their interaction with the bulk flow to be able to describe an evolving free surface film on a solid support. The necessary boundary conditions are discussed next.

\section{BOUNDARY CONDITIONS}

\section{A. Concentration}

For the concentration field the boundary conditions were discussed in connection with a purely diffusive transport for a system confined by rigid plates. ${ }^{73-75}$ Assuming the velocity is zero at the rigid substrate (no-slip condition, see Sec. III B) the conditions for the full model-H are similar. We have zero diffusive flux through the substrate $(z=0)$,

$$
\partial_{z}\left[\sigma_{c} \Delta c-\partial_{c} f(c)\right]=0
$$

and obtain in the general case an evolution equation for the concentration (see the Appendix)

$$
\partial_{t} c+\mathbf{v} \cdot \nabla c=-M^{-}\left[-\sigma_{c} \partial_{z} c-\sigma^{-} \Delta_{\|} c+\partial_{c} f^{-}(c)\right],
$$

where $\Delta_{\|}=\nabla_{\|} \cdot \nabla_{\|}$and $\nabla_{\|}=\left(\partial_{x}, \partial_{y}\right)$. Here, however, we will focus on a surface energy that (i) does not depend on con- centration gradients $\left(\sigma^{-}=0\right)$ and (ii) relaxes instantaneously to its equilibrium value $\left(M^{-} \rightarrow \infty\right)$.

At the free surface $[z=h(x, y, t)]$ one has the condition of zero diffusive flux through the moving surface, i.e., $\mathbf{n} \cdot \mathbf{j}_{\rho_{1}}=0$ with $\mathbf{j}_{\rho_{1}}$ as defined in Eqs. (10) and (38),

$$
\mathbf{n}=\frac{\left(-\partial_{x} h,-\partial_{y} h, 1\right)}{\left[1+\left(\partial_{x} h\right)^{2}+\left(\partial_{y} h\right)^{2}\right]^{1 / 2}}
$$

is the normal vector of the free surface. The change from the total flux $\mathbf{j}_{\rho_{1}}^{\prime}$ [Eq. (9)] to $\mathbf{j}_{\rho_{1}}=\mathbf{j}_{\rho_{1}}^{\prime}-\rho_{1} \mathbf{v}$ exactly accounts for the transformation into the frame moving locally with the surface. One gets

$$
\mathbf{n} \cdot \nabla\left[\sigma_{c} \Delta c-\partial_{c} f(c)\right]=0 .
$$

The second condition is in the general case again an evolution equation for the concentration field on the boundary as derived in the Appendix. The evolution equation is valid in the local comoving frame, i.e.,

$$
\partial_{t} c+\mathbf{v} \cdot \nabla c=-M^{+}\left[\sigma_{c}(\mathbf{n} \cdot \nabla) c-\sigma^{+} \Delta_{s} c+\partial_{c} f^{+}(c)\right],
$$

where $\Delta_{s}=\nabla_{s} \cdot \nabla_{s}$, and the surface nabla operator is defined as $\nabla_{s}=(\mathbf{I}-\mathbf{n n}) \cdot \nabla$. In the following we assume as above $\sigma^{+}=0$ and $M^{+} \rightarrow \infty$. We will drop the respective terms after the nondimensionalization in Sec. IV. Take note, that the units of the surface parameters differ from the ones of the corresponding bulk parameters: $\left[M^{ \pm}\right]=\mathrm{s} / \mathrm{kg},\left[\sigma_{s}^{ \pm}\right]=\mathrm{kg} \mathrm{m}^{2} / \mathrm{s}^{2}$, $\left[\partial_{c} f^{ \pm}\right]=\mathrm{N} / \mathrm{m}=\mathrm{kg} / \mathrm{s}^{2}$.

We finally point out, that the concept of an evolution equation for the concentration at the surface should not be confused with an evolution equation for a soluble surfactant that is located at the surface but is also in part dissolved in the bulk liquid. There, one normally assumes a certain rate for transitions of surfactant molecules from the surface to the bulk and vice versa. As a consequence, one then deals with separate transport equations for the species at the interface and in the bulk (see, e.g., Ref. 3). Even for finite $M^{+}$, Eq. (69) does not correspond to such a surfactant evolution equation but only describes the concentration at the surface with a finite relaxation time to equilibrium. $\mathrm{M}^{+}$quantifies the mismatch of surface relaxation and bulk relaxation time.

\section{B. Velocity}

The boundary conditions for the velocity fields are the no-slip and no-penetration condition at the solid substrate $(z=0)$,

$$
\mathbf{v}=0
$$

and the force equilibrium at the free surface $(z=h)$,

$$
\left(\underline{\boldsymbol{\tau}}-\underline{\boldsymbol{\tau}}_{\text {air }}\right) \cdot \mathbf{n}=-\gamma(c) \mathbf{n} \nabla \cdot \mathbf{n}+\nabla_{s} \gamma(c) .
$$

Note that $\nabla \cdot \mathbf{n}$ corresponds to the curvature of the free surface. We assume that the ambient air does not transmit any force $\left(\underline{\tau}_{\text {air }}=0\right)$. The first term on the rhs of Eq. (71) corresponds to the Laplace or curvature pressure, whereas the second one represents a Marangoni force tangential to the interface and results from the variation of the surface tension along the surface caused normally by a solutal or thermal 
Marangoni effect. As shown in the Appendix these terms can be derived from a minimization procedure.

For a pure Navier-Stokes problem the Marangoni term is often modeled as a linear dependence of the surface tension on concentration or temperature. Here, however, one has to use a condition in accordance with the interface energies introduced when discussing the boundary conditions for the concentration field, i.e., at Eq. (69). For $\sigma^{+}=0$ and $M^{+} \rightarrow \infty$ [see Eq. (69)] the surface tension $\gamma(c)$ corresponds to $f^{+}(c)$ plus a constant (reference tension $\gamma_{0}$, see below Sec. V). For $\sigma^{+} \neq 0$ the surface tension depends as well on concentration gradients $\gamma=\gamma\left[c,\left(\nabla_{s} c\right)^{2}\right]$, a concept that has not yet been followed in the literature. Considering a finite $M^{+}$would correspond to a $\gamma(c, t)$, i.e., to a dynamical surface tension characterized by a relaxation time towards its equilibrium value. Both complications will not be considered further in the present paper.

The boundary condition (71) is of vectorial character, i.e., three scalar conditions are derived by projecting it onto $\mathbf{n}, \mathbf{t}_{1}$, and $\mathbf{t}_{2}$, respectively, where

$$
\mathbf{t}_{1}=\frac{\left(1,0, \partial_{x} h\right)}{\left[1+\left(\partial_{x} h\right)^{2}\right]^{1 / 2}}, \quad \mathbf{t}_{2}=\frac{\left(0,1, \partial_{y} h\right)}{\left[1+\left(\partial_{y} h\right)^{2}\right]^{1 / 2}}
$$

are the (nonorthogonal) tangent vectors. The resulting scalar conditions

$$
\begin{aligned}
& -\sigma_{c}(\mathbf{n} \cdot \nabla c)^{2}-p_{\mathrm{eff}}+2 \eta \mathbf{n} \cdot(\nabla \mathbf{v}) \cdot \mathbf{n}=-\gamma(c) \nabla \cdot \mathbf{n}, \\
& -\sigma_{c}\left(\mathbf{t}_{1} \cdot \nabla c\right)(\mathbf{n} \cdot \nabla c)+\eta \mathbf{t}_{1} \cdot\left[\nabla \mathbf{v}+(\nabla \mathbf{v})^{T}\right] \cdot \mathbf{n}=\mathbf{t}_{1} \cdot \nabla \gamma(c),
\end{aligned}
$$

$-\sigma_{c}\left(\mathbf{t}_{2} \cdot \nabla c\right)(\mathbf{n} \cdot \nabla c)+\eta \mathbf{t}_{2} \cdot\left[\nabla \mathbf{v}+(\nabla \mathbf{v})^{T}\right] \cdot \mathbf{n}=\mathbf{t}_{2} \cdot \nabla \gamma(c)$

correspond to equilibria of normal and tangential forces, respectively.

At the free surface one has furthermore the kinematic condition, i.e., the prescription that the surface follows the flow field

$$
\partial_{t} h=\mathbf{n} \cdot \mathbf{v} \sqrt{1+\left(\nabla_{\|} h\right)^{2}}
$$

which can be written in a more compact form as

$$
\left(\partial_{t} \mathbf{h}\right) \cdot \mathbf{n}=\mathbf{v} \cdot \mathbf{n},
$$

where the vector $\mathbf{h}=h(x, y, t) \mathbf{e}_{z}$ is tracking the free surface.

\section{NONDIMENSIONALIZATION}

Next, we nondimensionalize the bulk equations and the boundary equations in two steps: (i) introduction of abstract scales for velocity, pressure, length, concentration, and energy density that leads us to a set of dimensionless numbers; (ii) introduction of problem specific length and velocity scales to obtain a minimal set of dimensionless numbers valid for the problems without external driving studied here.

\section{A. Abstract scales}

Introducing scales

$\begin{array}{ccc}\text { dimensionless } & \text { scale } & \text { dimensional } \\ t^{\prime} & \tau_{v}=l / U & t=\tau_{v} t^{\prime} \\ \mathbf{x}^{\prime} & l & \mathbf{x}=l \mathbf{x}^{\prime} \\ \mathbf{v}^{\prime} & U & \mathbf{v}=U \mathbf{v}^{\prime} \\ p^{\prime} & P & p=P p^{\prime} \\ c^{\prime} & C & c=C c^{\prime} \\ f^{\prime}\left(c^{\prime}\right) & E & f(c)=E f^{\prime}\left(c^{\prime}\right) \\ f^{\prime \pm}\left(c^{\prime}\right) & E^{ \pm} & f^{ \pm}(c)=E^{ \pm} f^{\prime \pm}\left(c^{\prime}\right)\end{array}$

one obtains after dropping the primes the dimensionless bulk equations

$$
\partial_{t} c+\mathbf{v} \cdot \nabla c=- \text { Ts } \nabla \cdot\left\{\nabla\left[\operatorname{Ko} \Delta c-\partial_{c} f(c)\right]\right\}
$$

and

$$
\operatorname{Ps}\left[\frac{\partial \mathbf{v}}{\partial t}+\mathbf{v} \cdot \nabla \mathbf{v}\right]=-\nabla \cdot\left\{\mathrm{Ko}^{\prime}(\nabla c)(\nabla c)+p_{\text {eff }-\mathbf{I}}\right\}+\frac{\operatorname{Ps}}{\operatorname{Re}} \Delta \mathbf{v},
$$

where

$$
p_{\text {eff }}=p-\mathrm{Ko}^{\prime}(c+1) \Delta c-\frac{1}{2} \mathrm{Ko}^{\prime}(\nabla c)^{2} .
$$

We defined the dimensionless numbers

Reynolds number $\operatorname{Re}=\frac{U l \rho}{\eta}$,

Korteweg number $1 \quad \mathrm{Ko}=\frac{\sigma_{c} C^{2}}{l^{2} E}$,

Korteweg number $2 \mathrm{Ko}^{\prime}=\frac{\sigma_{c} C^{2}}{l^{2} P}$,

Time scale ratio $\mathrm{Ts}=\frac{M E}{U l C^{2}}$,

Pressure scale ratio $\quad \mathrm{Ps}=\frac{\rho U^{2}}{P}$.

We propose the name "Korteweg number" because both of them are related to the Korteweg stresses. The Korteweg numbers can be seen as "bulk Marangoni numbers." For the determination of the energy scale $E$, see Sec. V.

The scaled boundary conditions for the concentration field at both interfaces are the no-flux condition

$$
0=\mathbf{n} \cdot \nabla\left[\operatorname{Ko} \Delta c-\partial_{c} f(c)\right]
$$

and the evolution equations for the concentration at the surface 


$$
\partial_{t} c+\mathbf{v} \cdot \nabla c=-\mathrm{Ts}^{ \pm}\left[\mathrm{Ko} \mathbf{n} \cdot \nabla c-\mathrm{Ko}^{ \pm} \Delta_{s} c+\operatorname{En}^{ \pm} \partial_{c} f^{ \pm}(c)\right] .
$$

For the substrate one sets $\mathbf{n}=(0,0,-1)$.

At the free surface the conditions for the normal and tangential forces are

$$
-\mathrm{Ko}^{\prime}(\mathbf{n} \cdot \nabla c)^{2}-p_{\mathrm{eff}}+2 \frac{\mathrm{Ps}}{\operatorname{Re}} \mathbf{n} \cdot(\nabla \mathbf{v}) \cdot \mathbf{n}=-S \gamma(c) \nabla \cdot \mathbf{n},
$$

$$
\begin{aligned}
& -\mathrm{Ko}^{\prime}\left(\mathbf{t}_{1} \cdot \nabla c\right)(\mathbf{n} \cdot \nabla c)+\frac{\mathrm{Ps}}{\operatorname{Re}} \mathbf{t}_{1} \cdot\left[\nabla \mathbf{v}+(\nabla \mathbf{v})^{T}\right] \cdot \mathbf{n} \\
& \quad=S \mathbf{t}_{1} \cdot \nabla \gamma(c), \\
& -\mathrm{Ko}^{\prime}\left(\mathbf{t}_{2} \cdot \nabla c\right)(\mathbf{n} \cdot \nabla c)+\frac{\mathrm{Ps}}{\mathrm{Re}} \mathbf{t}_{2} \cdot\left[\nabla \mathbf{v}+(\nabla \mathbf{v})^{T}\right] \cdot \mathbf{n} \\
& \quad=S \mathbf{t}_{2} \cdot \nabla \gamma(c),
\end{aligned}
$$

respectively, where $\gamma$ is the dimensionless surface tension referred to below in Sec. V as $\gamma^{\prime}$. The dimensionless numbers are either given above or listed next

Boundary Korteweg number $\mathrm{Ko}^{ \pm}=\frac{\sigma^{ \pm} C^{2}}{l^{3} E}$,

Boundary time scale ratio $\mathrm{Ts}^{ \pm}=\frac{E l^{2} M^{ \pm}}{U C^{2}}$,

Boundary energy number $\quad \operatorname{En}^{ \pm}=\frac{E^{ \pm}}{l E}$,

Surface tension number $S=\frac{\gamma_{0}}{l P}$.

\section{B. Specific scales}

For relaxational settings, i.e., systems without external driving forces, one might specify scales based on the "internal" diffusive or convective transport. Assuming very viscous liquids and taking into account that all structure formation will be driven by the decomposition process, it is convenient to base all scales on the diffusive processes only. Fixing $\mathrm{Ko}=1$ and $\mathrm{Ts}=1$ length and velocity scales become

$$
l=\sqrt{\frac{\sigma_{c}}{E}} C \text { and } \quad U=\frac{M E}{l C^{2}},
$$

respectively. Choosing a pressure scale based on the energy density scale

$$
P=E
$$

identifies the two Korteweg numbers, i.e., $\mathrm{Ko}^{\prime}=\mathrm{Ko}=1$. The specific forms of Reynolds and Pressure numbers are then

$$
\operatorname{Re}=\frac{M E \rho}{\eta C^{2}} \quad \text { and } \quad \operatorname{Ps}=\frac{\rho M^{2} E}{l^{2} C^{4}}=\frac{\rho M^{2} E^{2}}{\sigma_{c} C^{6}},
$$

respectively, i.e., $\mathrm{Ps} / \mathrm{Re}=\eta M / l^{2} C^{2}=\eta M E / \sigma_{c} C^{4}$. The dimensionless numbers related to the boundaries become

$$
\mathrm{Ts}^{ \pm}=\frac{l^{3} M^{ \pm}}{M}=\frac{M^{ \pm} \sigma_{c}^{3 / 2} C^{3}}{M E^{3 / 2}} \quad \text { and } \quad \mathrm{Ko}^{ \pm}=\frac{\sigma^{ \pm}}{l \sigma_{c}}=\frac{\sigma^{ \pm} E^{1 / 2}}{C \sigma_{c}^{3 / 2}} .
$$

Note that $\mathrm{Ts}^{ \pm} \rightarrow \infty$ for $M^{ \pm} \rightarrow \infty$ and $\mathrm{Ko}^{ \pm}=0$ for $\sigma^{ \pm}=0$ (see discussion in Sec. III),

$$
\operatorname{En}^{ \pm}=\frac{E^{ \pm}}{l E}=\frac{E^{ \pm}}{\sigma_{c}^{1 / 2} E^{1 / 2} C} \quad \text { and } \quad S=\frac{\gamma_{0}}{l E}=\frac{\gamma_{0}}{\sigma_{c}^{1 / 2} E^{1 / 2} C} .
$$

The relation of $S$ and the "classical" Marangoni number is discussed in the next section.

\section{LOCAL ENERGIES}

For the local bulk and surface energies we use a simple polynomial, an approximation valid near the critical point. However, it is straightforward to introduce other expressions derived using Flory-Huggins or more advanced theories. ${ }^{4}$ For the bulk energy a symmetric quartic potential is used

$$
f(c)=f_{0}-\frac{a(T)}{2} c^{2}+\frac{b}{4} c^{4}
$$

corresponding to the nondimensional form [Eqs. (78)]

$$
f^{\prime}\left(c^{\prime}\right)=\frac{1}{4}\left(c^{\prime 2}-1\right)^{2}+\text { const }
$$

with $E=b C^{4}$ and $C=\sqrt{a / b}$. Note that the physical constants appearing in the bulk can be measured experimentally or be computed from first principles. Indeed, as discussed in Ref. 56 in the context of a gas-liquid interface and in Ref. 109 for binary mixtures they can be derived from intermolecular potentials.

For the surface energies of the two interfaces we use the respective quadratic expressions

$$
f^{ \pm}(c)=\gamma_{0}^{ \pm}+\widetilde{a}^{ \pm} c+\frac{\tilde{b}^{ \pm}}{2} c^{2} .
$$

Note that in the framework of model-H for a film of binary mixture the surface energies $f^{-}(c)$ and $f^{+}(c)$ correspond to the concentration dependent surface tensions of the liquidsolid and the liquid-gas interface, respectively. This implies that $f^{+}(c)$ is responsible for a linear $\left(\tilde{b}^{+}=0\right)$ or nonlinear $\left(\tilde{b}^{+} \neq 0\right)$ Marangoni effect. The surface energies $\gamma_{0}^{+}$and $\gamma_{0}^{-}$ are the respective reference surface tensions at $c=0$.

Using the reference surface tension of the free surface as a scale for both interfaces, i.e., $E^{+}=E^{-}=\gamma_{0}^{+}=\gamma_{0}$, we arrive at the nondimensional expressions

$$
f^{\prime \pm}\left(c^{\prime}\right)=\frac{\gamma_{0}^{ \pm}}{\gamma_{0}^{+}}+a^{ \pm} c^{\prime}+\frac{b^{ \pm}}{2} c^{\prime 2}
$$

and identify $\gamma^{\prime}=f^{\prime+}\left(c^{\prime}\right)$. The dimensionless parameters 


$$
a^{ \pm}=\frac{\widetilde{a}^{ \pm} C}{\gamma_{0}^{+}} \quad \text { and } \quad b^{ \pm}=\frac{\widetilde{b}^{ \pm} C^{2}}{\gamma_{0}^{+}}
$$

describe preferential adsorption of one of the species at the interface and changes in the interaction between the species at the respective interfaces. Inspecting Eq. (86) it becomes clear that the "classical" Marangoni number for a linear Marangoni effect is $\mathrm{Ma}=a^{+} S$. The corresponding number for a quadratic Marangoni effect is $\mathrm{Ma}_{2}=b^{+} S$ (compare, for instance, Refs. 110 and 111). Furthermore, we can now specify

$$
\mathrm{En}^{+}=\mathrm{En}^{-}=\frac{\gamma_{0}}{l E}=S
$$

This implies that the boundary conditions for concentration and momentum transport at the free surface are intrinsically coupled. Note, finally that these considerations only apply for $\mathrm{Ts}^{ \pm} \rightarrow \infty$ and $\mathrm{Ko}^{ \pm}=0$. See discussion in Secs. III and IV. In the following we only work with dimensionless quantities and drop all primes.

We are now equipped with a complete model to investigate a wide variety of systems involving decomposing mixtures with free surfaces. Although, the boundary conditions in Sec. III are written for a film on a solid substrate they can be easily adapted for free standing films, i.e., for a film with two free surfaces. Also droplets of a mixture on a solid substrate can be studied if the given model is supplemented by a condition at the contact line such as a concentration dependent equilibrium contact angle. This will be the scope of future work.

To understand the evolution of the surface and concentration profiles of a decomposing film we next analyze (i) the homogeneous and vertically stratified base state solutions, (ii) the transversal instability of the base state solutions that lead to the experimentally observed film profiles and concentration patterns, and (iii) the full nonlinear time evolution. Part (i) will be studied in the remainder of the present paper, part (ii) forms the content of a sequel planned to appear in 2008, and part (iii) will be presented in future work.

\section{BASE STATES}

The understanding of the behavior of a thin film of a mixture on a solid substrate has to be based on an analysis of the base state solutions. For a film on a horizontal substrate without further driving forces parallel to the substrate the base states are quiescent, i.e., the velocity of the fluid mixture is zero. We distinguish two types of quiescent base states: (a) completely homogeneous flat film and (b) horizontally (transversally) homogeneous but vertically stratified film.

\section{A. Completely homogeneous film}

A completely homogeneous film of arbitrary thickness $h(x, y)=h_{0}$, with arbitrary concentration $c(\mathbf{x})=c_{0}$ and with quiescent fluid $\mathbf{v}_{0}=0$ corresponds to a base state solution of the systems (79)-(97) only if there exists no energetic bias at the solid-liquid interface or the free surface, i.e., without any linear or nonlinear Marangoni effect: $a^{ \pm}=b^{ \pm}=0$. The corresponding effective pressure is $p_{\text {eff }}=0$.

For energetically biased interfaces the boundary conditions for the concentration field are only fulfilled if $\partial_{c} f^{ \pm}\left(c_{0}\right)$ $=0$, i.e., for $c_{0}=-a^{+} / b^{+}=-a^{-} / b^{-}$. For finite $a^{+}, a^{-}$this is from the experimental point of view a very unlikely case. Here we will not pursue it further. However, for $a^{+}=a^{-}=0$ a homogeneous film of a critical mixture $\left(c_{0}=0\right)$ represents a base state for any $b^{+}$and $b^{-}$. This case corresponds to a purely quadratic Marangoni effect. Experimentally, it is not a very common case but was studied in hydrodynamics for films of alcohol solutions ${ }^{111}$ and also as a problem of purely diffusive demixing in a gap. The latter case was analyzed in detail in Refs. 74 and 75, and will serve as a benchmark for our linear stability analysis in the forthcoming sequel paper.

\section{B. Vertically stratified, horizontally homogeneous film}

Depositing a thin film of a mixture on a solid substrate it is to be expected that processes that lead to a vertical stratification are much faster than processes that lead to a horizontal structuring if the film thickness is similar or below the length scale of bulk decomposition. The vertically stratified films may on a larger time scale undergo a further horizontal structuring. The finally emerging horizontal length scales and structures can be understood from the "short-time" vertical layering. Therefore we focus next on a systematic investigation of steady layered films.

A flat layer $\left(h=h_{0}\right)$ of a quiescent fluid mixture $\left(\mathbf{v}_{0}=0\right)$ represents a base state if the vertical concentration profile $c$ $=c_{0}(z)$ is a steady solution of the classical one-dimensional nonconvective Cahn-Hilliard equation

$$
\partial_{t} c=\partial_{z z}\left[\partial_{z z} c-\partial_{c} f(c)\right]
$$

and the boundary conditions (i) $0=\partial_{z}\left[\partial_{z z} c_{0}-\partial_{c} f\left(c_{0}\right)\right]$ (at $z=0$ and $z=h$ ) and (ii) $0=\left[ \pm \partial_{z} c_{0}+S \partial_{c} f^{ \pm}\left(c_{0}\right)\right]$ ("- - at $z=0$ and "+" at $z=h$ ). Taking into account (i) one has to solve the bulk equation $\partial_{z z} c_{0}-\partial_{c} f\left(c_{0}\right)+K_{1}=0$ with boundary conditions (ii). The constant of integration $K_{1}$ represents the dimensionless chemical potential for an inhomogeneous equilibrium as discussed after Eq. (57).

The remaining equations and boundary conditions are fulfilled with $p_{\text {eff }}=p_{\text {eff }}(z)=-\left(\partial_{z} c_{0}\right)^{2}+$ const, i.e., the layers are completely characterized by $c_{0}(z)$. The condition corresponds to an exact compensation of the vertical component of the Korteweg force $\nabla \cdot(\nabla c)(\nabla c)$ by the vertical component of the pressure gradient $\nabla p_{\text {eff }}$. The horizontal component is identically zero.

In the following we determine families of solutions in terms of concentration profiles for (i) energetically neutral or nonbiased surfaces, (ii) symmetrically biased surfaces, (iii) antisymmetrically biased surfaces, and (iv) asymmetrically biased surfaces. Thereby we characterize the concentration profiles by the energy

$$
E=f^{+}+f^{-}+\int_{0}^{h}\left[\left(\partial_{z} c\right)^{2}+f(c)\right] d z-h f(1),
$$

i.e., their relative energy w.r.t. the state of minimal energy $(c= \pm 1)$, and the $L_{2}$-norm, 

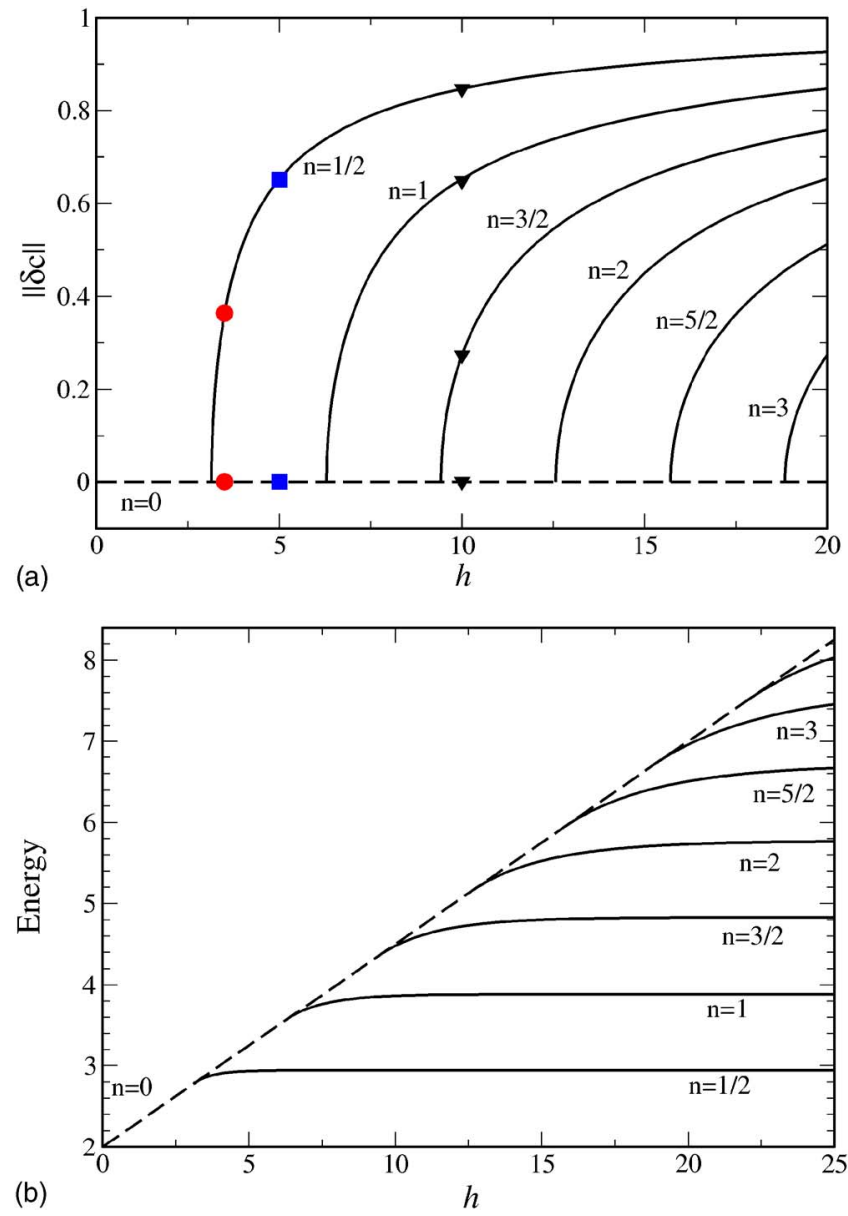

FIG. 1. (Color online) Branches of steady vertical concentration profiles for energetically nonbiased (neutral) surfaces $\left(a^{ \pm}=b^{ \pm}=0\right)$ and a critical mixture $(\bar{c}=0)$ is dependent on the film thickness $h$. Shown are (a) the $L_{2}$-norm and (b) the energy $E$. Dashed line: trivial homogeneous solution. Selected corresponding profiles are given in Fig. 2. $S=1$, and the symbols are explained in the main text.

$$
\|\delta c\|=\sqrt{\frac{1}{h} \int_{0}^{h}[c(z)-\bar{c}]^{2} d z,}
$$

where $\bar{c}$ is the mean concentration. Note that $E$ should only be used to compare films of identical $h$ and $\bar{c}$. Although the profiles $c(z)$ can be expressed analytically through Jacobi elliptic functions (see Ref. 112 and also the Appendix of Ref. 113), determination using numerical continuation techniques ${ }^{114}$ is more practical.

\section{Energetically neutral surfaces}

The results for the trivial case of energetically neutral solid substrate and free film surface are given in Fig. 1 for a critical mixture, i.e., the case of zero mean concentration $\bar{c}$ $=0$. Shown are the $L_{2}$-norm and the energy $E$ per film area. Figure 2 presents selected concentration profiles. The base states for a film correspond to selected solutions of the onedimensional bulk Cahn-Hilliard equation. A multiple of the period has to be equal to the film thickness. At the substrate and the free surface the profile always has a local extrema. This allows us to classify the obtained solution branches by

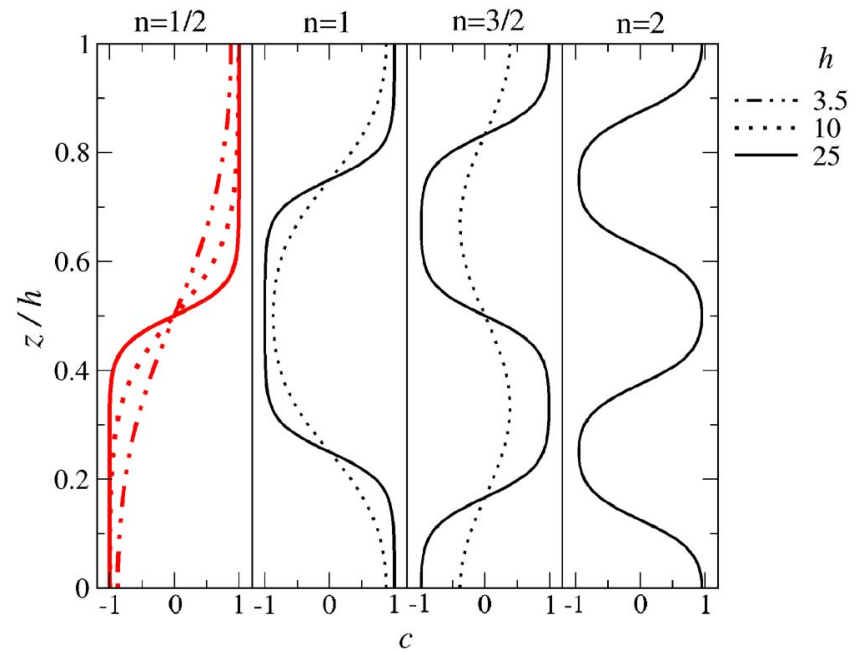

FIG. 2. (Color online) Selected concentration profiles corresponding to Fig. 1. Note that each of the solutions has a "twin" obtained by $c \rightarrow-c$ that also corresponds to an allowed profile. This degeneracy may be lifted by energetically biased surfaces (depending on the symmetry $z \rightarrow h_{0}-z$, see below). The $n=1 / 2$ profiles corresponding to the energy minimum for the respective film thickness are shown as heavy (red online) lines.

the number of periods $n$. The simplest stratified films correspond to half a period $(n=1 / 2)$, one period $(n=1)$, one and a half periods $(n=3 / 2)$, and so on.

Note that the solutions with an integer $n$ are symmetric with respect to a reflection at the plane $z=h_{0} / 2$, i.e., $c_{0}(z)=c_{0}\left(h_{0}-z\right)$. We call them in the following " $z$-reflection-symmetric." They are accompanied by a twin solution with identical $L_{2}$-norm and energy obtained by an inversion of concentration: $c_{0}(z) \rightarrow-c_{0}(z)$. On the contrary, the solutions with a noninteger $n$ are antisymmetric with respect to a reflection at the plane $z=h_{0} / 2$, i.e., $c_{0}(z)=-c_{0}\left(h_{0}\right.$ $-z$ ) ("z-reflection-antisymmetric"). The resulting second solution has naturally identical $L_{2}$-norm and energy, and can also be obtained by an inversion of concentration. We will also call it the twin solution.

The bifurcation diagram is not very involved. For all film thicknesses there exists the trivial homogeneous solution with $\|\delta c\|=0$ and linearly increasing energy $E=2+h / 4$ (broken lines in Fig. 1). Nontrivial branches bifurcate successively at $h_{i}=i \pi / k_{c}$, where $i=1,2,3, \ldots$ and $k_{c}=\sqrt{-\partial_{c c} f\left(c_{0}\right)}$ $=\sqrt{1-3 c_{0}^{2}}$ corresponds to the critical wavenumber for the linear instability of the homogeneous solution $c=c_{0}$ of Eq. (100). For the critical mixture considered in Fig. 1 one finds $h_{i}=i \pi$. Furthermore, all characteristics like $E_{i}[h]$ or $\|\delta c\|_{i}[h]$ of all branches $n=i$ with $i \geq 1$ can be mapped onto the characteristics of the $n=1 / 2$ branch. For example, for the energy one has $E_{i}[h]=E_{1 / 2}[h /(2 i)]$. Note, however, that the bifurcations are degenerate because as discussed above two twin solutions related by symmetry bifurcate at once.

A thin film in an experiment will tend towards the constellation with the minimal energy [see Figs. 1(b) and 2], i.e., for $h<\pi$ the homogeneous layer and for $h>\pi$ the stratified layer with $n=1 / 2$. The multilayer constellations with $n \geq 1$ may, however, appear as transients as they are saddle fixed points in phase space that attract time evolutions from a certain basin of attraction and repel them consecutively into the 

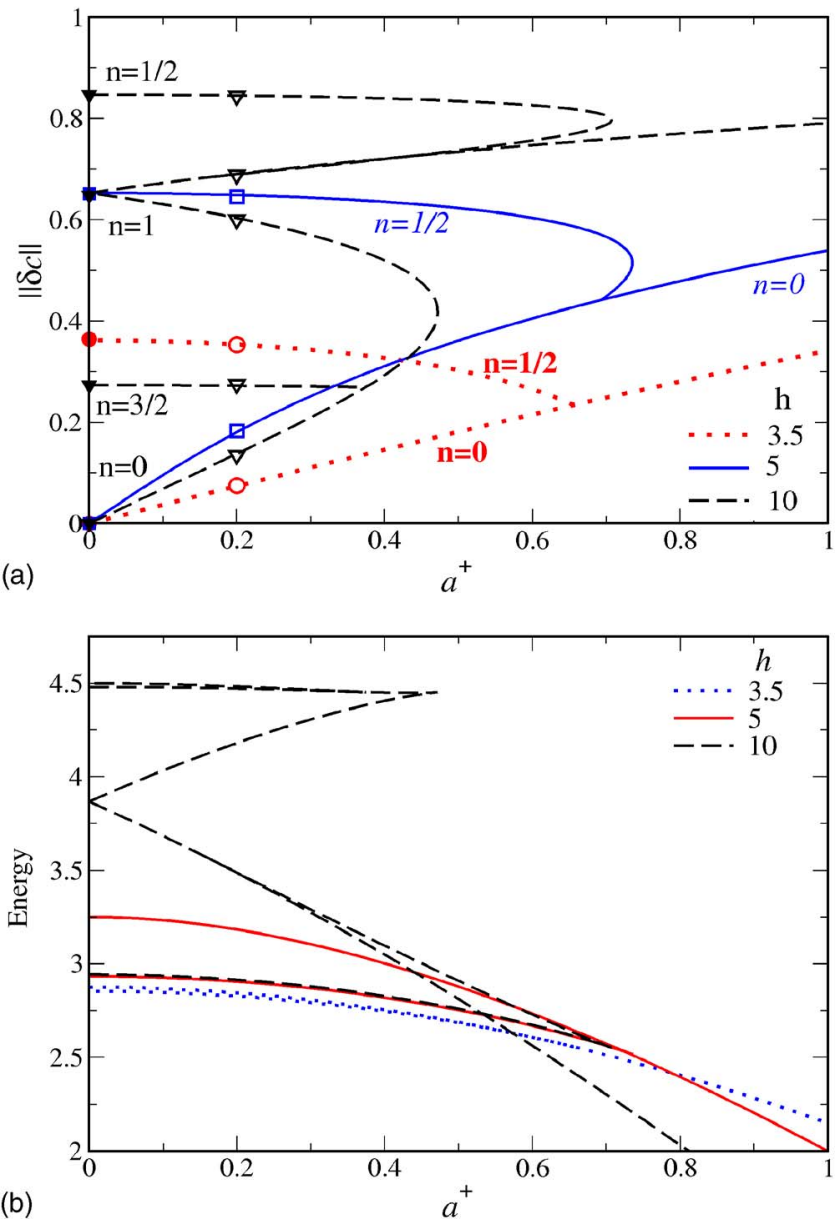

FIG. 3. (Color online) Branches of steady vertical concentration profiles for linearly symmetrically biased surfaces $\left(a^{+}=a^{-}, b^{ \pm}=0\right)$ of a critical mixture $(\bar{c}=0)$ dependent of bias $a$ for film thicknesses as given in the legend. Shown are (a) the $L_{2}$-norm and (b) the energy $E$. Selected corresponding profiles are given in Fig. 5. The branch numbers $n$ for the different film thicknesses are given using different fonts: $h=3.5$ bold, $h=5$ italic, $h=10$ normal. Lines and labels are of corresponding colors (online). $S=1$, and the symbols are explained in the main text.

few unstable directions (for a more extensive discussion of that concept in connection with dewetting on heterogeneous substrates, see Ref. 49).

\section{Symmetrically biased surfaces}

The presented rather detailed description of the steady states for energetically neutral surfaces will help us to understand the involved behavior for biased surfaces. Allowing for arbitrary linear $\left(a^{-}, a^{+}\right)$and quadratic $\left(b^{-}, b^{+}\right)$energetic biases opens a four-dimensional parameter space additionally to the parameter "film thickness." We give an overview of the system behavior by focusing on a linear bias $\left(b^{-}=b^{+}\right.$ $=0$ ), and by using several special ratios $a^{+} / a^{-}$. In this way we obtain a 2D parameter space spanned by $a^{+}$and $h$.

In the present section we assume that the two surfaces energetically prefer the same component with equal strength $\left(a^{+}=a^{-}\right)$, i.e., we have symmetrically biased surfaces. Figures 3 and 4 show characteristics of solution branches dependent of the bias for fixed film thickness and dependent of the film thickness for fixed bias, respectively. Corresponding so-
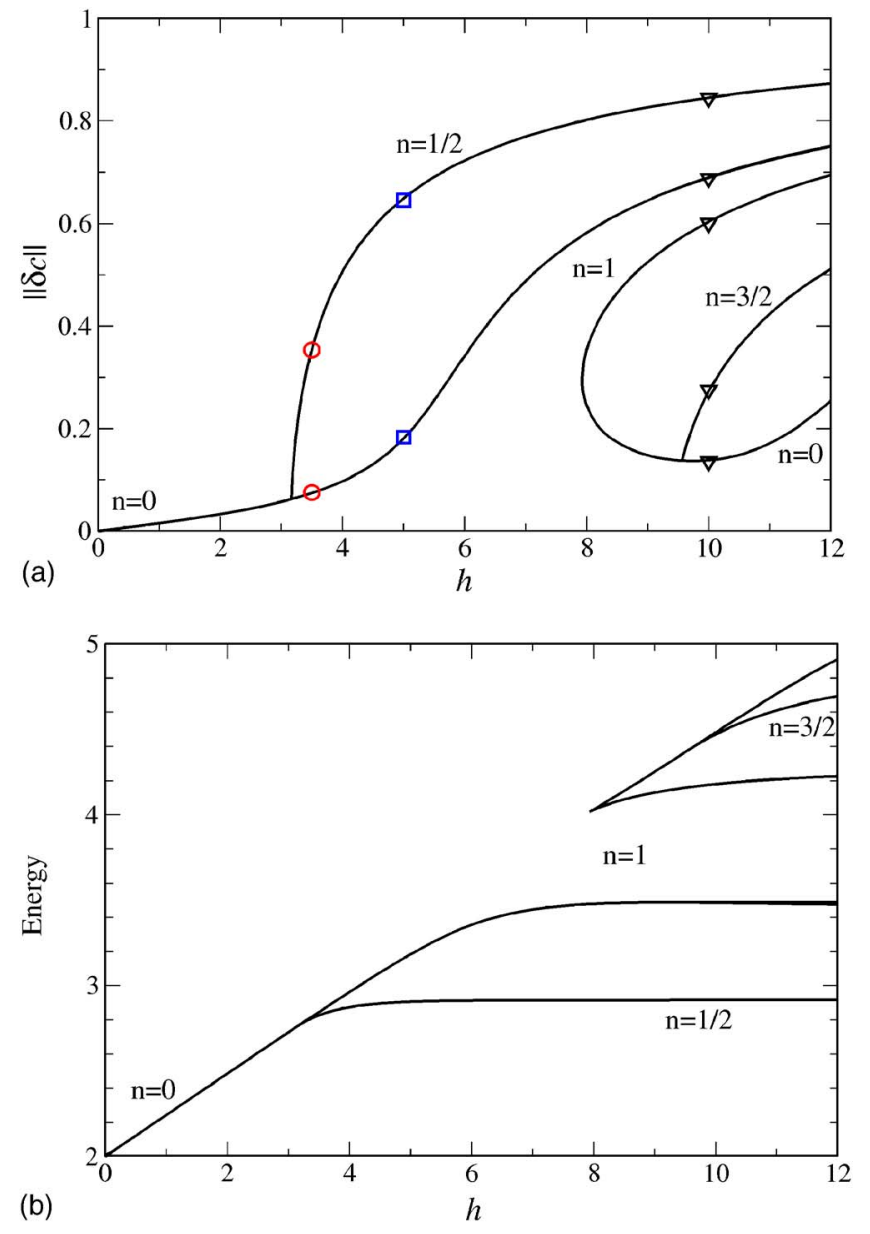

FIG. 4. (Color online) Branches of steady vertical concentration profiles dependent of film thicknesses for linearly symmetrically biased surfaces $\left(a^{+}=a^{-}=0.2, b^{ \pm}=0\right)$ and a critical mixture $(\bar{c}=0)$. Shown are (a) the $L_{2}$-norm and (b) the energy $E$. Selected corresponding profiles are given in Fig. 5. $S=1$, and the symbols are explained in the main text.

lutions between Figs. 1 and 3 are marked by filled symbols in the L2-norm plots. Hollow symbols indicate correspondences between Figs. 3 and 4. Concentration profiles for $a^{+}$ $=0.2$ corresponding to the hollow symbols are given in Fig. $5(\mathrm{a})$, whereas panel (b) gives profiles for a large bias of $a^{+}=0.6$.

First, we focus on Fig. 3. We introduce branch names indicating the "nonbiased branch" (Fig. 1) they are emerging from. This convention does not correspond to actual properties of the concentration profile. For instance, the profiles on the $n=0$ branch in Fig. 3 are not homogeneous any more. For small film thicknesses $\pi<h<2 \pi$ only two solutions exist at $a^{+}=0$ corresponding to two branches for increasing $a^{+}>0$. Thereby the $n=0[n=1 / 2]$ branch is unstable [stable]. Further increasing the bias the two branches approach each other. For $h=3.5$ the $n=1 / 2$ branch terminates in a supercritical bifurcation on the $n=0$ branch. For $h=5$ the stable $n=1 / 2$ branch first undergoes a saddle-node bifurcation turning unstable before it finally terminates in a subcritical bifurcation on the $n=0$ branch. Beyond the bifurcation the $n=0$ branch is stable in both cases.

For $i \pi<h<(i+1) \pi$ one finds $i$ solutions at $a^{+}=0$ exemplified in Fig. 3 for $h=10$, where four solutions exist. In- 

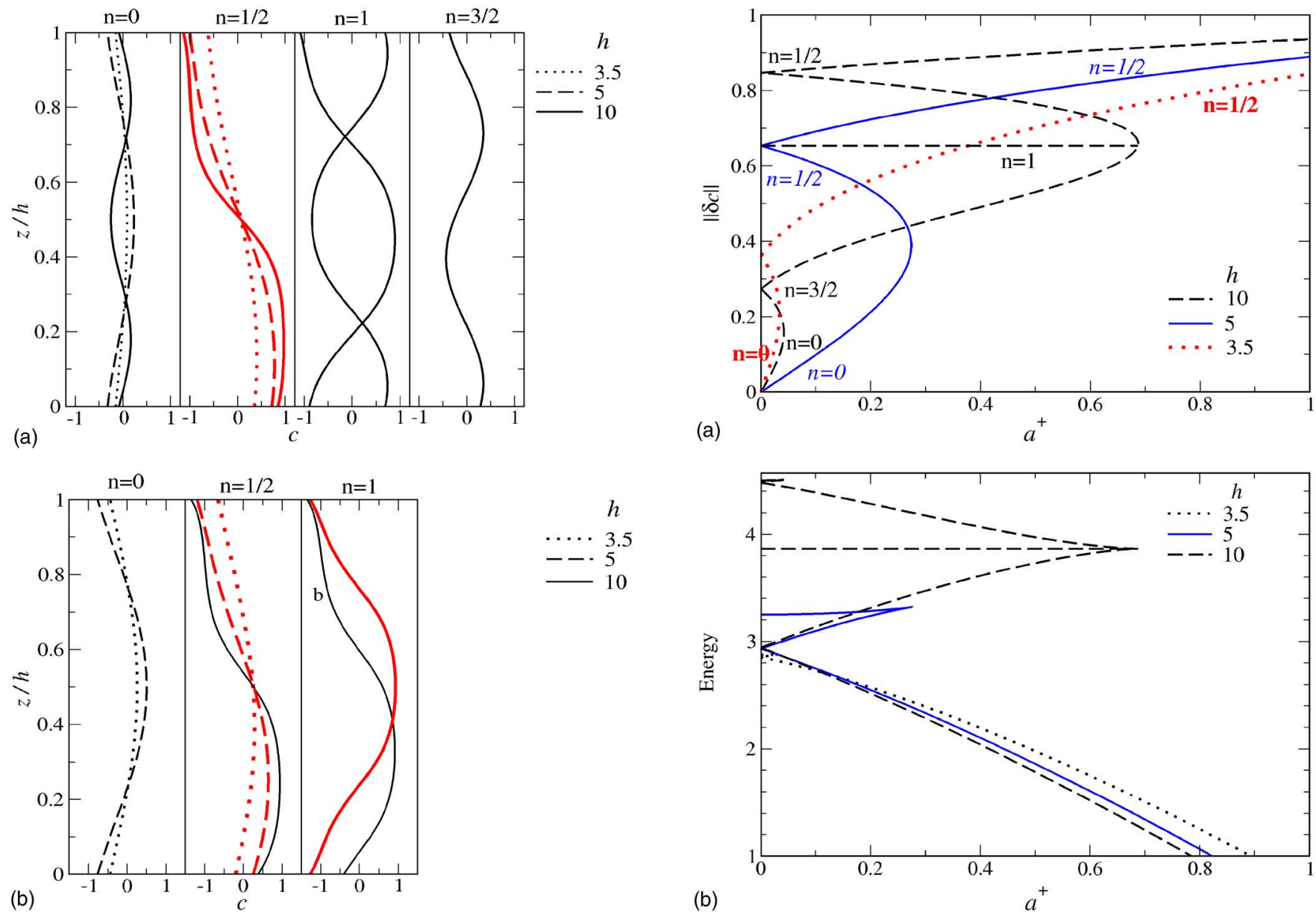

FIG. 5. (Color online) Selected concentration profiles corresponding to Figs. 3 and 4 for (a) $a^{+}=0.2$ and (b) $a^{+}=0.6$ sorted by branch number as indicated in Fig. 4. Film thicknesses are indicated in the legends. The profiles corresponding to the energy minimum for the respective film thickness and bias are shown as heavy (red online) lines. The minuscule $\mathrm{b}$ in the $n=1$ panel for $a^{+}=0.6$ denotes the profile on the side branch of the $n=1$ branch (cf. Fig. 3).

creasing $a^{+}>0$ one finds, however, five emerging branches because the degeneracy of the $n=1$ solution at $a^{+}=0$ is lifted by the energetic bias. See the discussion of symmetries above in Sec. VI B 1.

The $z$-reflection-symmetric solutions (integer $n$ ) have at $a^{+}=0$ a "twin"-solution obtained by $c(z) \rightarrow-c(z)$ that reacts differently when imposing a symmetric energetic bias, i.e., the bias lifts the degeneracy and two distinct branches are generated like, for instance, in Fig. 3 for $h=10$ and $n=1$ (see also profiles in Fig. 5). Note that the $n=0$ branch is a special case without degeneracy at $a^{+}=0$ (trivial solution at $a^{+}=0$ ). On the contrary, the "twin"-solutions of the $z$-reflection-antisymmetric solutions (noninteger $n$ ) do not react in a different way to a symmetric bias, i.e., their degeneracy is not lifted.

Coming back to the case $h=10$ we see that when further increasing $a^{+}$most branches end in bifurcations. One branch finally survives for large bias corresponding to a layer of "liquid +" enclosed by two layers of "liquid -" that is preferred by both surfaces for $a^{+}>0$. This implies that depending on the strength of bias the energetic minimum corresponds to qualitatively different stratifications: bilayer

FIG. 6. (Color online) Branches of steady vertical concentration profiles for linearly antisymmetrically biased surfaces $\left(a^{+}=-a^{-}, b^{ \pm}=0\right)$ and a critical mixture $(\bar{c}=0)$ dependent of bias $a$ for film thicknesses as given in the legend. Shown are (a) the $L_{2}$-norm and (b) the energy $E$. Selected corresponding profiles are given in Fig. 7. $S=1$. The branch numbers $n$ for the different film thicknesses are given in different fonts: $h=3.5$ bold, $h=5$ italic, $h=10$ normal. Lines and labels are of corresponding colors online.

$(n=1 / 2)$ structure for small $a^{+}$and a sandwich trilayer $(n=1)$ structure for large $a^{+}$(see heavy red online) lines in Fig. 5.

Note finally that the diagram is symmetric w.r.t. $a^{+} \rightarrow-a^{+}$. Focusing on the branch of lowest energy that represents the solutions selected by the system we see that the role is taken for small (large) $a^{+}$by the $n=1 / 2(n=1$ or $n$ $=0$ ) branch. It is intuitively clear that a strong symmetric bias will suppress the $z$-reflection-antisymmetric solutions. The alternative view of fixing $a^{+}$and changing $h$ is given for $a^{+}=0.2$ in Fig. 4 allowing for a better comparison with Fig. 1. From this representation it becomes clear that for $a^{+}>0$ the two branches emerging from the $n=1$ solution "break off" the $n=0$ branch at $h=2 \pi$. A similar process occurs at all $h=2 i \pi$ for integer $i$.

\section{Antisymmetrically biased surfaces}

In contrast to the preceding section, here we assume $a^{+}=-a^{-}$, i.e., the two surfaces energetically prefer different components. The preference is, however, equally strong. We focus on $a^{+}>0$, i.e., the free surface prefers the $c<0$ com- 


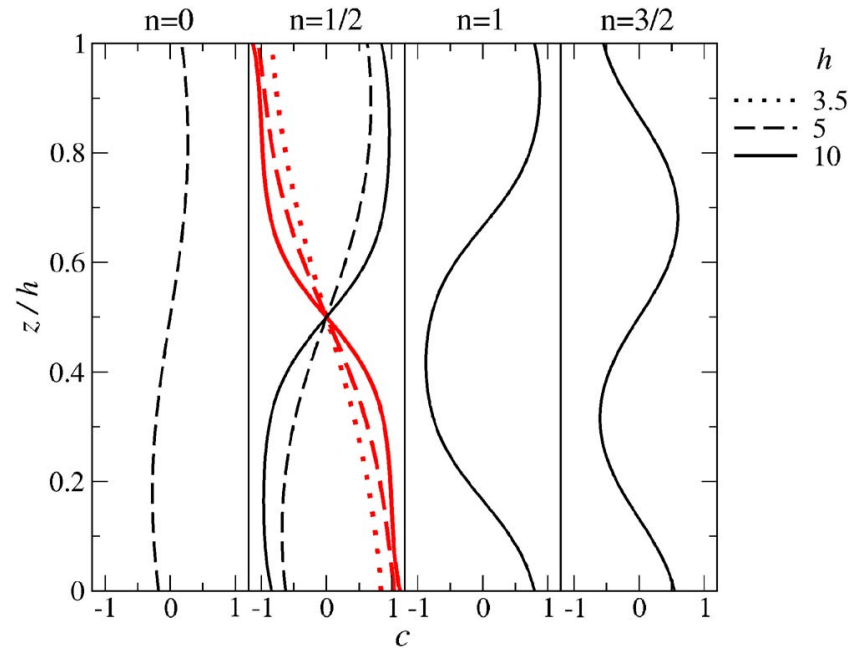

FIG. 7. (Color online) Selected concentration profiles corresponding to Fig. 6 for $a^{+}=0.2$ sorted by branch number as indicated in Fig. 6. Film thicknesses are indicated in the legend. The profiles corresponding to the energy minimum for the respective film thickness and bias are shown as heavy (red online) lines.

ponent. The case $a^{+}<0$ is related by symmetry. Figures 6 and 8 show solution branches in dependence of the bias and of film thickness, respectively (in analogy to Figs. 3 and 4). Selected corresponding profiles are given in Fig. 7.

In contrast to the case of symmetrically biased surfaces we find that for small $a^{+}>0$ two branches emerge from the $n=1 / 2$ and $n=3 / 2$ solutions but only one from the $n=1$ solution. Here, the degeneracy of the solutions at $a^{+}=0$ is only lifted for the $z$-reflection-antisymmetric solutions (noninteger $n$ ), but not for the $z$-reflection-symmetric solutions (integer $n$ ). In the former case one of the twin solutions at $a^{+}=0$ is favored by the antisymmetric bias, whereas the other one is disfavored, i.e., they decrease and increase their energy with $a^{+}$, respectively [Fig. 6(b)]. One of the $n=1 / 2$ solutions is the only one that "survives" for large bias $a^{+}$. It is furthermore this solution that corresponds to the energy minimum for all $a^{+}$[see heavy (red online) lines in Fig. 7].

The alternative view of fixing $a^{+}$and changing $h$ is given for $a^{+}=0.2$ in Fig. 8. Contrary to Sec. VI B 2 for $a^{+}>0$ the $n=0$ branch is "broken off" at $h=2 n \pi$ by the respective two branches emerging from the noninteger $n$ solutions.

\section{Asymmetrically biased surfaces}

As an intermediate case compared to the two preceding sections, we focus next on $a^{-}=0$ and $a^{+}>0$, i.e., the substrate is energetically neutral, whereas the free surface prefers the $c<0$ component. Note that the cases $a^{-}=0$ and $a^{+}<0, a^{-}>0$ and $a^{+}=0, a^{-}<0$ and $a^{+}=0$ are related by symmetry.

Figures 9 and 10 show solution branches in dependence of the bias for fixed film thickness and dependent of the film thickness for fixed bias, respectively. Here the bias lifts all degeneracies existing for $a^{+}=0$, i.e., from each solution at $a^{+}=0$ emerge two branches (beside the $n=0$ branch). Corre-
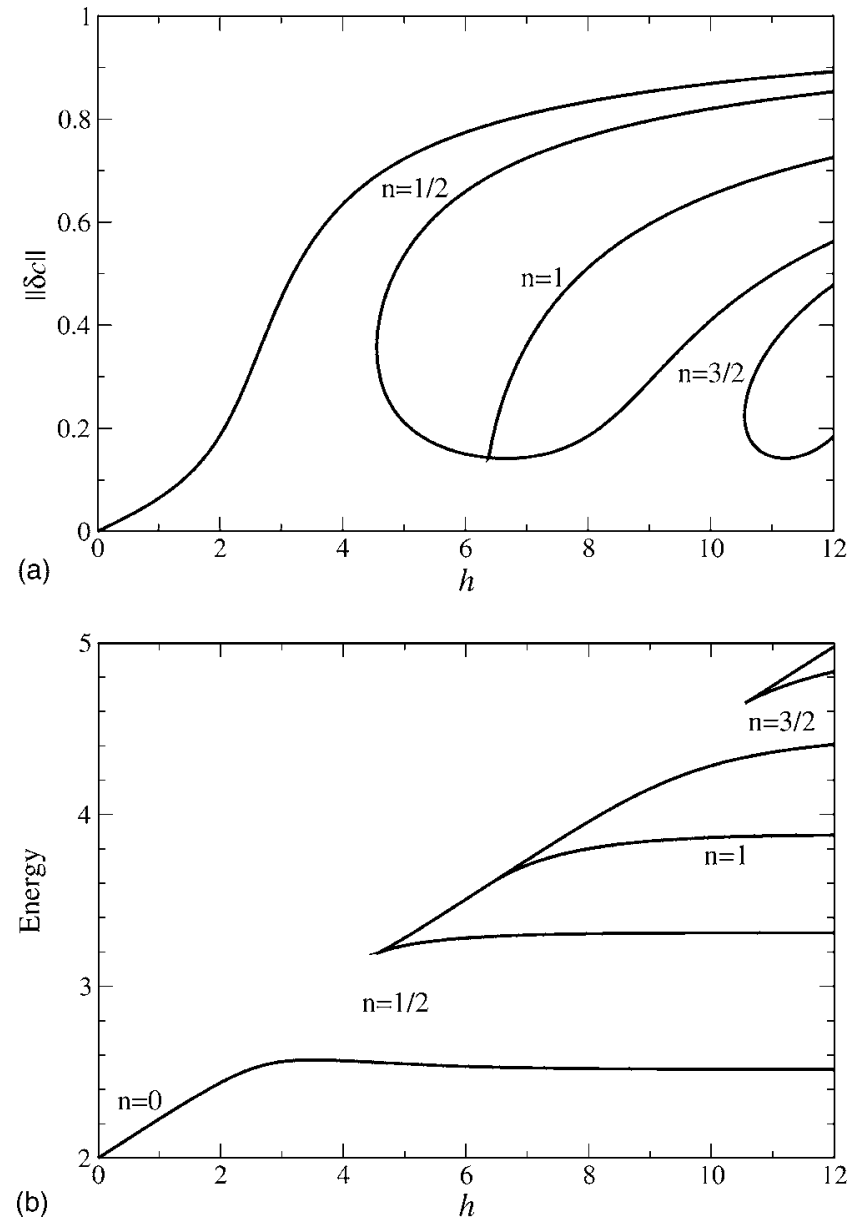

FIG. 8. Branches of steady vertical concentration profiles for linearly antisymmetrically biased surfaces $\left(a^{+}=-a^{-}=0.2, b^{ \pm}=0\right)$ and a critical mixture $(\bar{c}=0)$ dependent of film thickness. Shown are (a) the $L_{2}$-norm and (b) the energy $E$. Selected corresponding profiles are given in Fig. 7. $S=1$.

spondingly, Fig. 10 shows that for $a^{+}>0$ the two branches emerging from every integer and noninteger $n$ solution "break off" the $n=0$ branch at all $h=2 n \pi$.

The branch of lowest energy is here for all $a^{+}$and $h$ the $n=1 / 2$ branch, i.e., a simple two layer structure. This is, however, by no means a general result but depends on the specific asymmetry chosen. For a strong bias that is only slightly asymmetric (like, for instance, $a^{-}=a^{+}+\Delta$ with $\left.\Delta \ll a^{+}\right)$the branch of lowest energy will still be the sandwich structure discussed in Sec. VI B 2.

\section{Off-critical mixtures}

We have seen that the case of a critical mixture $\bar{c}=0$ can be used to understand the basic solution structure for stratified films. However, it has to be kept in mind that a critical mixture represents a rather special case. Experimental systems will normally consist of off-critical mixtures with $\bar{c} \neq 0$. In the present section we give selected results for the general case.

For small $\bar{c} \neq 0$ the branch structure for nonbiased surfaces is given in Fig. 11 for $\bar{c}=0.25$. It is qualitatively equivalent to the one for a critical mixture (cf. Fig. 1). For larger $\bar{c}$ the primary bifurcations become subcritical. Eventu- 

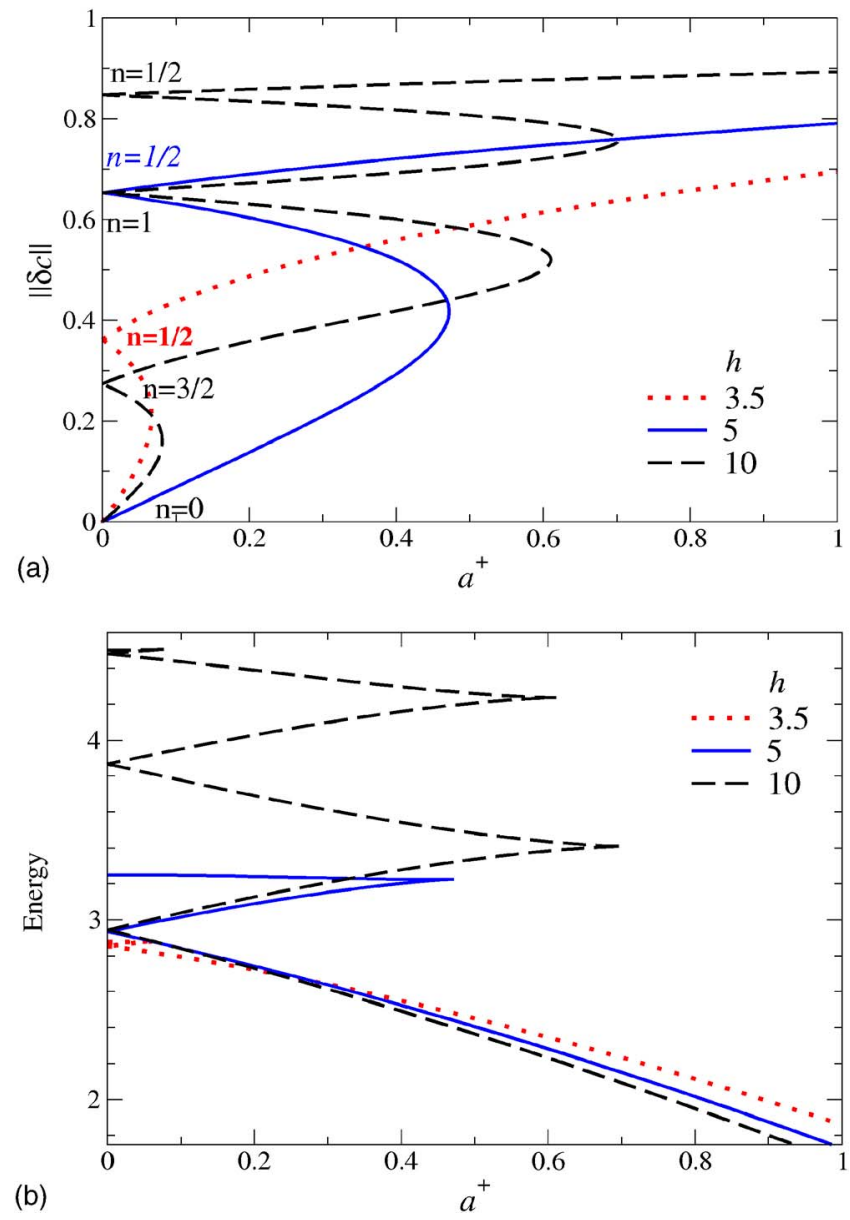

FIG. 9. (Color online) Branches of steady vertical concentration profiles for linearly asymmetrically biased surfaces $\left(a^{+}>0, a^{-}=b^{ \pm}=0\right)$ and a critical mixture $(\bar{c}=0)$ dependent of bias $a^{+}$for film thicknesses as given in the legend. Shown are (a) the $L_{2}$-norm and (b) the energy E. $S=1$. The branch numbers $n$ for the different film thicknesses are given in different fonts: $h$ $=3.5$ bold, $h=5$ italic, $h=10$ normal. Lines and labels are of corresponding colors online.

ally the trivial solution becomes linearly stable. It is, however, metastable, because finite perturbations may trigger a nonlinear instability. For those $\bar{c}$ no primary bifurcations exist. All branches of stratified solutions continue towards infinite thickness. The bifurcation diagrams for biased surfaces become quite involved for the subcritical and metastable case and will be discussed elsewhere.

Focusing on the case of supercritical primary bifurcations shown in Fig. 11 we next discuss the influence of symmetrically biased surfaces presented in Fig. 12. The general form of the bifurcation diagram for $a^{+}>0$ is qualitatively very similar to the case of a critical mixture (Fig. 3). However, the symmetry w.r.t. $a^{+} \rightarrow-a^{+}$does not hold anymore. It is replaced by a symmetry w.r.t. $\left(\bar{c}, a^{+}\right) \rightarrow\left(-\bar{c},-a^{+}\right)$.

Also for antisymmetrically biased surfaces one finds similar bifurcation diagrams for nonsymmetric (Fig. 13) and critical (Fig. 6) mixtures. The antisymmetry of the boundary conditions implies that the symmetry w.r.t. $a^{+} \rightarrow-a^{+}$also holds for the off-critical mixture. Finally, in the asymmetrically biased case shown in Fig. 14 all degeneracies at
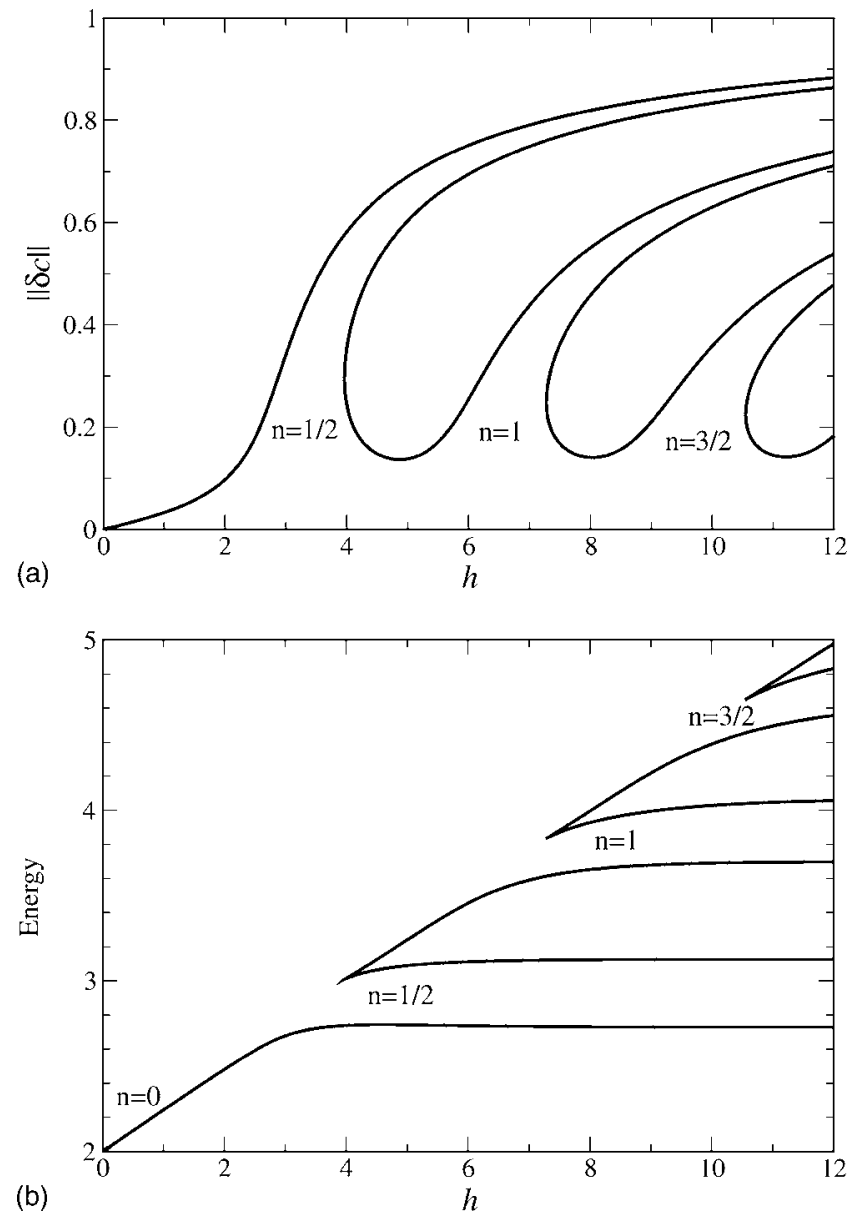

FIG. 10. Branches of steady vertical concentration profiles for linearly asymmetrically biased surfaces $\left(a^{+}=0.2, a^{-}=0, b^{ \pm}=0\right)$ and a critical mixture $(\bar{c}=0)$ dependent of film thicknesses. Shown are (a) the $L_{2}$-norm and (b) the energy $E . S=1$.

$a^{+}=0$ are broken as in the case of a critical mixture (Fig. 9). Furthermore, now also the symmetry w.r.t. $a^{+} \rightarrow-a^{+}$is broken.

\section{CONCLUSION}

We have proposed a dynamical model that describes the coupled decomposition and profile evolution of a free surface film of a binary mixture, a process frequently encountered in coating and structuring processes. An example is a thin film of a polymer blend on a solid substrate undergoing simultaneous phase separation and dewetting. We have based our approach on model-H coupling transport of the mass of one component (convective Cahn-Hilliard equation) and momentum (Navier-Stokes-Korteweg equations). We have used the framework of phenomenological nonequilibrium thermodynamics to derive a generalized model-H coupling transport equation for momentum, density, and entropy in the framework of phenomenological nonequilibrium thermodynamics. Then we have discussed the individual contributions representing, for instance, an internal Soret effect and interface viscosity. The model has been simplified for isothermal setting, vanishing interface viscosity, and internal energies re- 

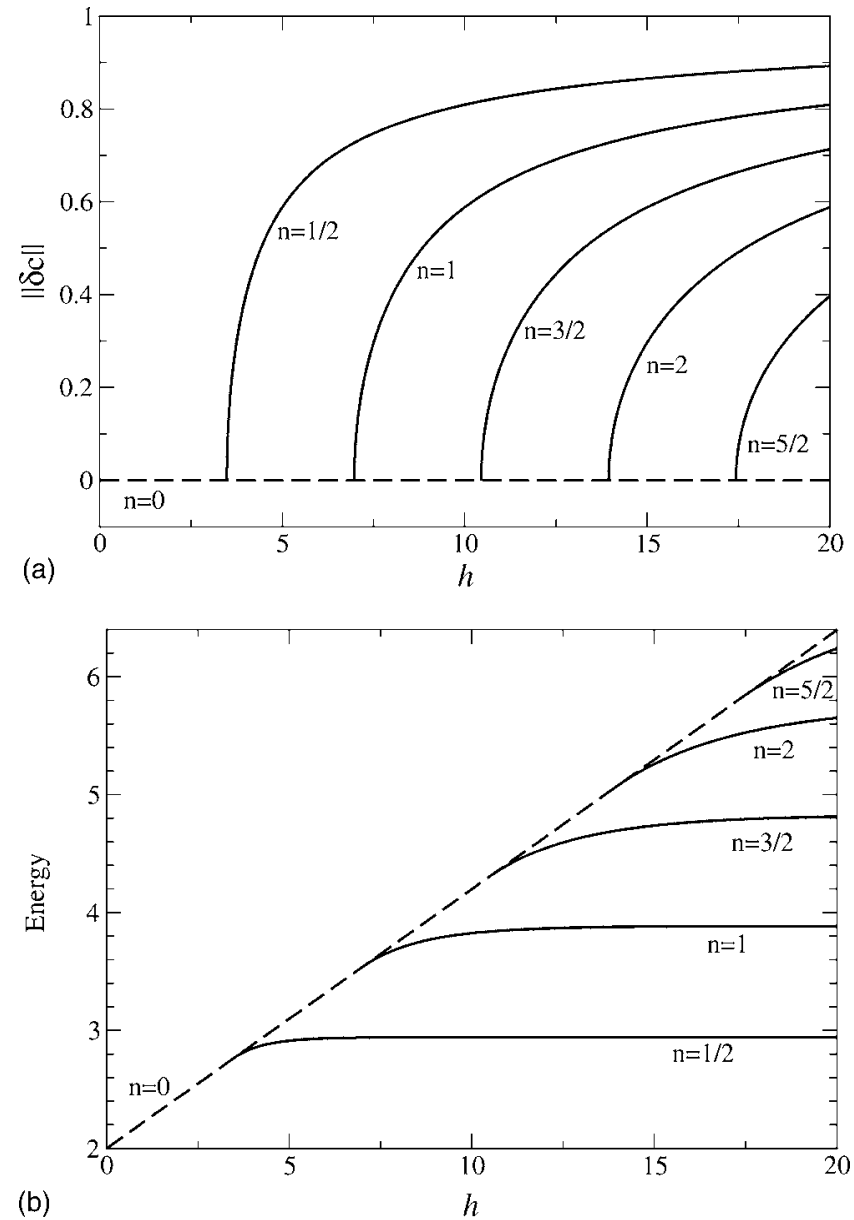

(b)

$h$

FIG. 11. Branches of steady vertical concentration profiles for nonbiased surfaces $\left(a^{ \pm}=b^{ \pm}=0\right)$ and off-critical mixtures $(\bar{c}=0.25)$ dependent of film thickness $h$. Shown are (a) the $L_{2}$-norm and (b) the energy $E$. $S=1$.

sulting from a setting close to the critical point of demixing. A comparison with literature results has clarified the issue of defining pressure and chemical potential.

To facilitate the description of a free surface profile we have introduced boundary conditions at the solid substrate and the free interface. It has been explained that the incorporation of hydrodynamic flow even in the case of extremely slow creeping flow is a necessary precondition for the description of evolving surface deflections. It has been shown that the dimensionless numbers entering the boundary conditions for the Cahn-Hilliard and the Korteweg-NavierStokes are closely related. Therefore they cannot by any means be chosen independently of each other. After nondimensionalization we have analyzed possible steady base state solutions for laterally homogeneous films of decomposing mixtures. In doing so we have distinguished vertically homogeneous and vertically stratified films. It has been shown that a plethora of stratified solutions exist that can be mapped, ordered and understood using continuation techniques and symmetry arguments. The obtained systematics will form the basis for the analysis of the lateral stability of the base states undertaken in the forthcoming part II. In the Appendix we have used variational calculus to independently confirm the boundary conditions for the static limiting case.

Our results on vertical stratifications can be compared to
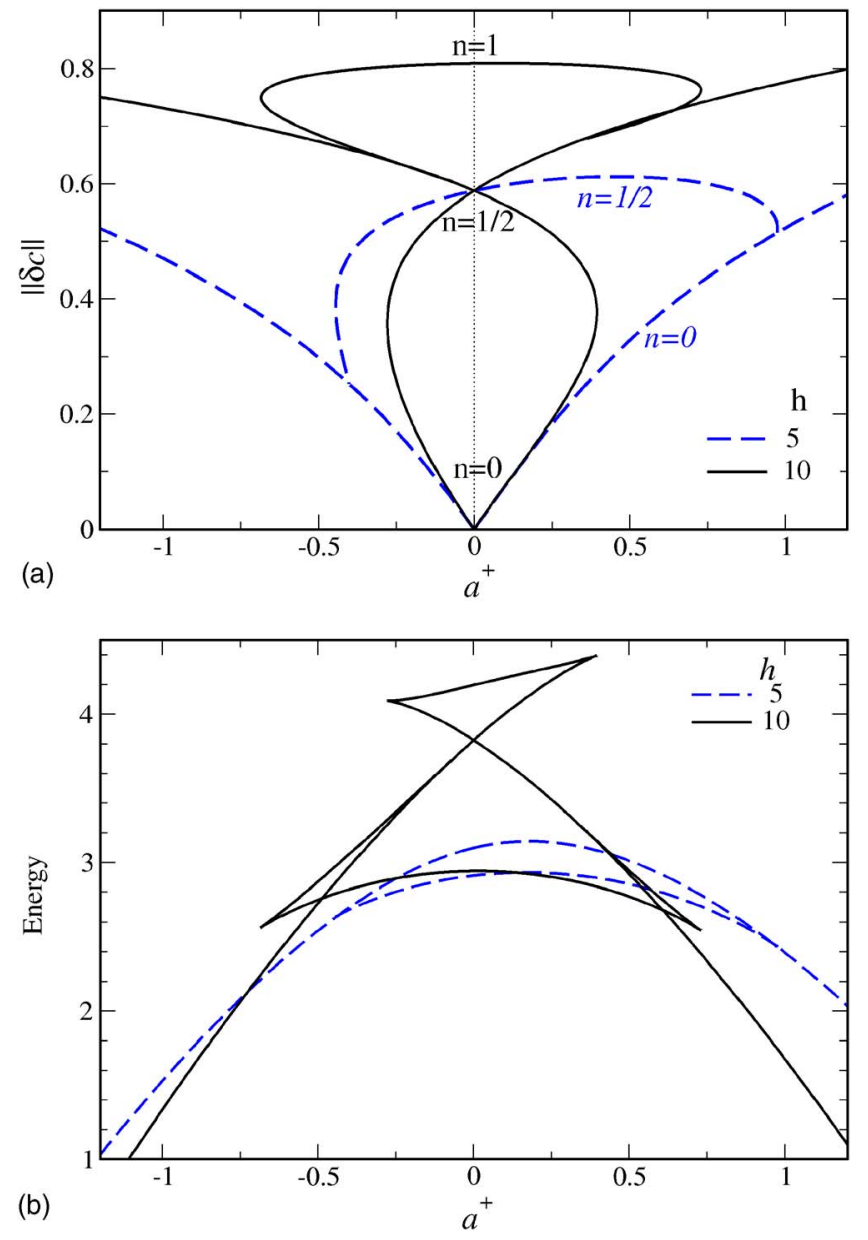

FIG. 12. (Color online) Branches of steady vertical concentration profiles for linearly symmetrically biased surfaces $\left(a^{+}=a^{-}, b^{ \pm}=0\right)$ and an off-critical mixture $(\bar{c}=0.25)$ dependent of bias $a^{+}$for film thicknesses as given in the legend. Shown are (a) the $L_{2}$-norm and (b) the energy $E$. $S=1$. The branch numbers $n$ for the different film thicknesses are given in different fonts: $h$ $=5$ italic, $h=10$ normal. Lines and labels are of corresponding colors online.

a broad variety of experimental data on static film structures. However, they can also be used to interpret transitions observed in slow time evolutions. Most of the results on vertical layering reviewed in Ref. 4 can be explained at least qualitatively. Most static vertical layerings observed in thin films of polymer blends either correspond to two-layer or sandwich-like three-layer structures that we have found to be the only structures of lowest energy depending on the energetic bias of the surfaces.

The interesting case of the evolution of a relatively thick $(500 \mathrm{~nm})$ decomposing d-PMMA/SAN blend film ${ }^{70}$ is presented in Fig. 16 of Ref. 4. The vertical profile develops from a homogeneous film to a two-layer structure, with d-PMMA collecting at the solid substrate. However, the path to equilibrium passes through a sandwich-like three-layer structure. This can be easily understood from the solution structure presented for antisymmetrically biased surfaces in Fig. 6 . There the $n=1$ (sandwich-like three-layer structure) has a lower energy than the $n=0$ or $n=3 / 2$ solution but a higher energy than the final $n=1 / 2$ solution. The $n=1$ solution corresponds to a saddle in phase space, i.e., it attracts time evolutions of a broad range of initial conditions and then expels 

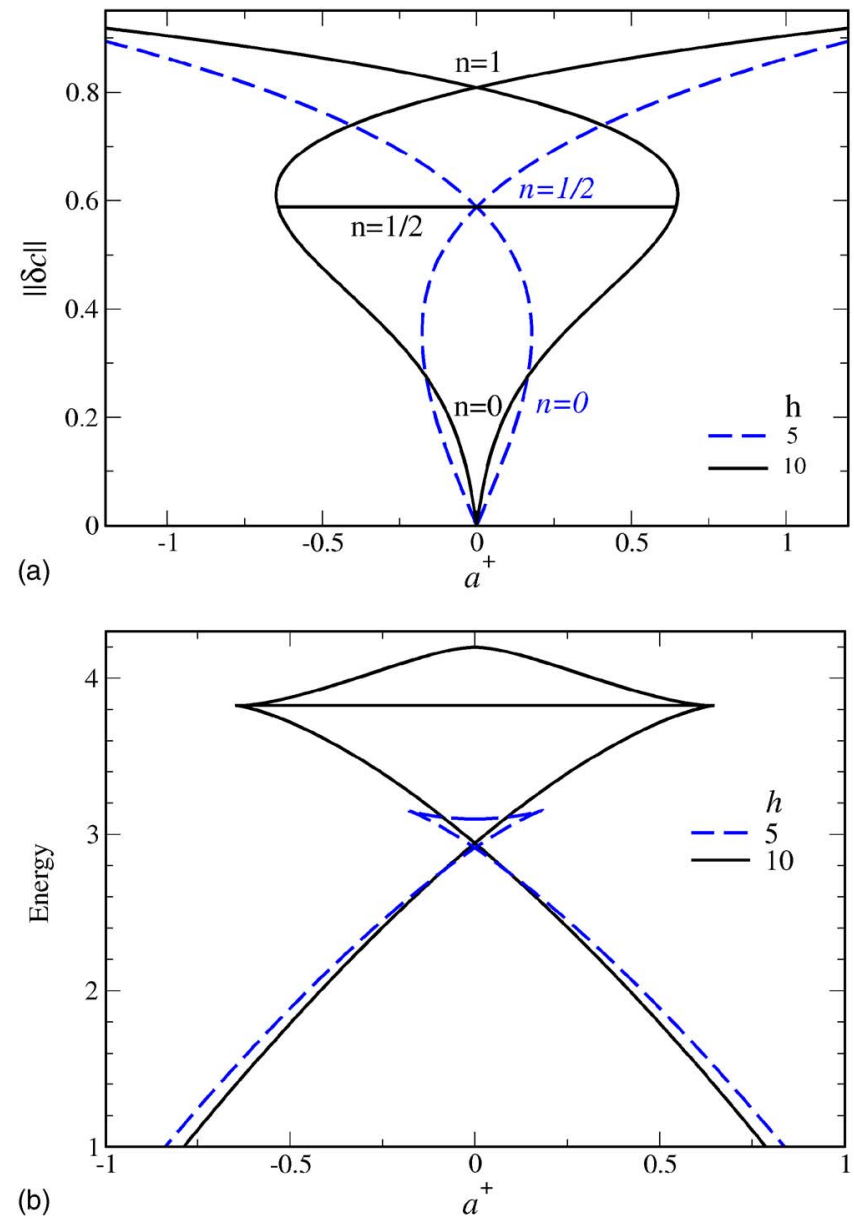

FIG. 13. (Color online) Branches of steady vertical concentration profiles for linearly antisymmetrically biased surfaces $\left(a^{+}=-a^{-}, b^{ \pm}=0\right)$ and an offcritical mixture $(\bar{c}=0.25)$ dependent of bias $a^{+}$for film thicknesses as given in the legend. Shown are (a) the $L_{2}$-norm and (b) the energy E. $S=1$. The branch numbers $n$ for the different film thicknesses are given in different fonts: $h=5$ italic, $h=10$ normal. Lines and labels are of corresponding colors online.

evolutions in its only unstable direction directing the evolution towards the final two-layer structure. Which "saddle solutions" will be involved in a time evolution depends on the wavelength of the fastest linear mode. For a similar discussion for dewetting films on a heterogeneous substrate, see Ref. 49 Sec. III.

In another experiment using a PEP/d-PEP blend film ${ }^{69}$ presented in Fig. 21 of Ref. 4 it is shown that the equilibrium layer structure changes from d-PEP/PEP/d-PEP to d-PEP/ PEP by varying the substrate surface energy. This corresponds in our idealized setting to a transition from symmetrically biased surfaces (e.g., at $h=10, a^{+}=0.8, n=1$ branch) to anti- or asymmetrically biased surfaces (e.g., at $h=10, a^{+}$ $=0.8, n=1 / 2$ branch). The observed change in the layering of lowest energy corresponds well to the experiments.

To conclude, the present work has presented a complete model to investigate a wide variety of systems involving the coupled evolution of surface and concentration profiles of free surface films of a decomposing mixture. It can be used to analyze vertically stratified but horizontally homogeneous films and their evolution in time. This includes layer inver-
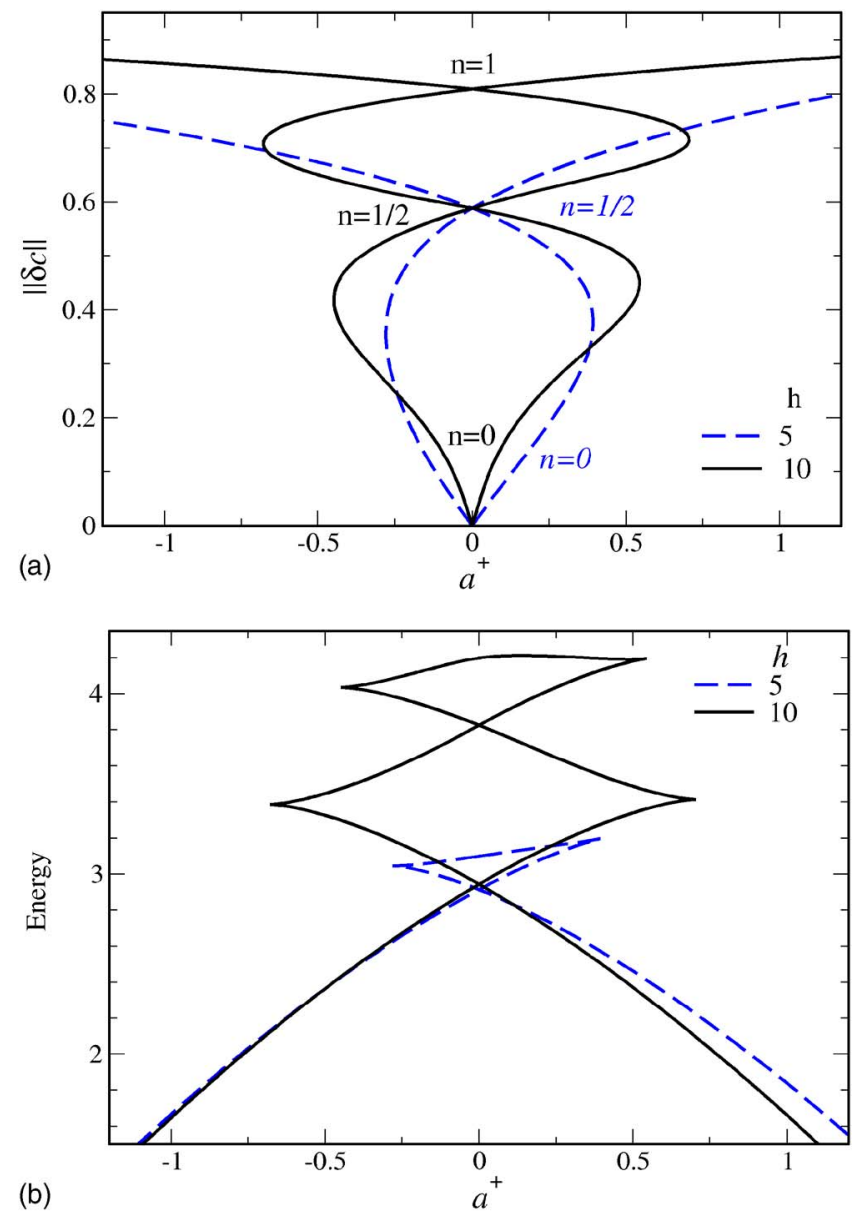

FIG. 14. (Color online) Branches of steady vertical concentration profiles for linearly asymmetrically biased surfaces $\left(a^{+} \neq 0, a^{-}=0, b^{ \pm}=0\right)$ and an off-critical mixture $(\bar{c}=0.25)$ dependent of bias $a^{+}$for film thicknesses as given in the legend. Shown are (a) the $L_{2}$-norm and (b) the energy $E$. $S=1$. The branch numbers $n$ for the different film thicknesses are given in different fonts: $h=5$ italic, $h=10$ normal. Lines and labels are of corresponding colors online.

sions of two-layer systems with diffuse or sharp interfaces that cannot be described by two-layer models for immiscible liquids. ${ }^{40-44}$ The dynamics of such an inversion is quite complex. For instance, for a substrate/liquid 1/liquid 2/air twolayer structure it involves the transient formation of drops of liquid 1 at the liquid 2/air interface. ${ }^{59}$ The typical distance of those droplets can now be calculated using a linear transversal stability analysis of the unstable two-layer profile. For antisymmetrically biased surfaces it exists, for instance, for $h=5$ up to $a^{+} \approx 0.3$ (Fig. 6).

Our model also allows us to study the emergence of permanent lateral structures using a transversal stability analysis of the stratified layers (see sequel paper) or a simulation in time. Especially, it allows to quantify the influence of hydrodynamic transport on the dynamics.

Note that the model can be adapted for several related problems. Although, the boundary conditions in Sec. III are posed for a film on a solid substrate they can easily be adapted for free standing films, i.e., for a film with two free surfaces. Also drops of a mixture on a solid substrate can be studied if the presented model is supplemented by a condition at the contact line. The latter will be the scope of future 
work. Also the used model of local bulk and interface energies can easily be replaced by more realistic functions as discussed in Ref. 4. Choosing parameters that correspond to a stable mixture also the dynamics of mixing in a free surface film can be studied.

\section{ACKNOWLEDGMENTS}

This work was supported by the European Union and Deutsche Forschungsgemeinschaft under Grants Nos. MRTN-CT-2004-005728 and SFB 486 B13, respectively. U. T. thanks MPIPKS and Peter Hänggi for support at various stages of the project. L. F. acknowledges support by the Israel Science Foundation under Grant No. 55/02.

\section{APPENDIX: VARIATIONAL APPROACH}

This Appendix uses variational calculus to derive the static limit of the bulk equations and boundary conditions from the underlying Helmholtz free energy functional of the Cahn-Hilliard-type ${ }^{72}$ for a two-dimensional film of binary mixture. The three-dimensional case will be presented elsewhere for a more general setting. The free energy functional

$$
F[c(x, z), h(x)]=F_{b}[c(x, z), h(x)]+F_{s}[c(x, h(x)), h(x)]
$$

consists of a bulk part $F_{b}$ and a surface part $F_{s}$ defined as

$$
\begin{aligned}
F_{b}= & \int_{-\infty}^{\infty} \int_{0}^{h(x)}\left[\frac{\sigma_{c}}{2}(\nabla c)^{2}+f(c)\right] d z d x-\lambda_{d} \\
& \times\left[\int_{\Omega} c_{1} d A-C_{1} A\right]-\lambda\left[\int_{\Omega} d A-A\right], \\
F_{s}= & \oint_{\partial \Omega} f_{s}(c) d s .
\end{aligned}
$$

The second and third integral in $F_{b}$ are taken over the same area as the first one, the symbol $\Omega$ is introduced for convenience. The symbol $\partial \Omega$ denotes the boundary of the domain of integration $\Omega$. We assume that the surface free energy does not depend on $(\nabla c)^{2}$, i.e., the bulk free energy gradient term is not amended at the free surface. Such a contribution and its physical consequences will be discussed elsewhere. The Lagrange multipliers $\lambda_{d}$ and $\lambda$ enforce mean concentration of component $1, c_{1}=(c+1) / 2$, and total area of the domain to have the prescribed values $C_{1}$ and $A$, respectively. Later, we will discuss their relation to the local chemical potentials $\mu_{2}, \mu_{d}$ and mechanical pressure $p$.

To vary $F$ with respect to all possible degrees of freedom in a transparent way we define small changes of the functions $c$ and $h$ as

$$
\begin{aligned}
& h(x ; \alpha)=h(x)+\alpha \zeta(x), \\
& c(x, z ; \alpha)=c(\mathbf{x} ; \alpha)=c(\mathbf{x})+\alpha \eta(\mathbf{x}),
\end{aligned}
$$

where $\zeta$ and $\eta$ are arbitrary admissible functions and $\mathbf{x}=(x, z)$. The variation of $h$ and $c$ corresponds to the operation $\left.\partial_{\alpha}\right|_{\alpha=0}$, i.e., $\delta h(x)=\zeta(x), \delta c(\mathbf{x})=\eta(\mathbf{x})$. However, not only the local concentration and the interface position are varied.
Varying the latter also implies that fluid elements have to vary their position due to convective motion.

The variation of the bulk contribution to the free energy functional can be written as

$$
\begin{aligned}
\delta F_{b}= & \int_{-\infty}^{\infty} \int_{0}^{h(x)}\left[\sigma_{c} \nabla c \cdot \nabla \eta+\left(\partial_{c} f-\frac{\lambda_{d}}{2}\right) \eta\right] d z d x \\
& -\delta \lambda_{d}\left[\int_{\Omega} c_{1} d A-C_{1} A\right]-\delta \lambda\left[\int_{\Omega} d A-A\right] \\
& +\int_{-\infty}^{\infty}\left[\frac{\sigma_{c}}{2}(\nabla c)^{2}+f(c)-\lambda_{d} c_{1}-\lambda\right] \zeta(x) d x,
\end{aligned}
$$

where we used $\delta \nabla c=\nabla \delta c$. Integrating by parts the term containing $\nabla \eta$, we get

$$
\begin{aligned}
\delta F_{b}= & \int_{-\infty}^{\infty} \int_{0}^{h(x)}\left[-\sigma_{c}(\Delta c) \eta+\left(\partial_{c} f-\frac{\lambda_{d}}{2}\right) \eta\right] d z d x \\
& -\delta \lambda_{d}\left[\int_{\Omega} c_{1} d A-C_{1} A\right]-\delta \lambda\left[\int_{\Omega} d A-A\right] \\
& +\oint_{\partial \Omega} \sigma_{c}(\nabla c) \cdot \mathbf{n} \eta d s \\
& +\int_{-\infty}^{\infty}\left[\frac{\sigma_{c}}{2}(\nabla c)^{2}+f(c)-\lambda_{d} c_{1}-\lambda\right] \zeta(x) d x .
\end{aligned}
$$

Next, we turn our attention to the surface contribution $F_{s}$. concentrating on the most interesting top part of the boundary $\partial \Omega$, i.e., the free surface. We denote the corresponding part of $F_{s}$ as $F_{s}^{\text {top }}$ and write it as

$$
F_{s}^{\mathrm{top}}=\int_{-\infty}^{\infty} f_{s}\left[c\left(\mathbf{x}_{s}\right)\right] \frac{d s}{d x} d x,
$$

where

$$
\frac{d s}{d x}=\sqrt{1+\left[\partial_{x} h(x)\right]^{2}} .
$$

For the point $\mathbf{x}_{s}(x)=[x, h(x)]$ at the free surface and its variation we have

$$
\begin{aligned}
& \mathbf{x}_{s}(x ; \alpha)=[x, h(x)+\alpha \zeta(x)], \\
& \delta \mathbf{x}_{s}(x)=[0, \zeta(x)] .
\end{aligned}
$$

Then $\delta c\left[\mathbf{x}_{s}(x)\right]=\nabla c\left(\mathbf{x}_{s}\right) \cdot \delta \mathbf{x}_{s}+\eta\left(\mathbf{x}_{s}\right)$. Using Eqs. (A9) and $\mathbf{x}_{s}(x)$ we have expressed the integrand of (A8) as a function of $x$. The variation of $F_{s}^{\text {top }}$ is

$$
\begin{aligned}
\delta F_{s}^{\mathrm{top}} & =\int_{-\infty}^{\infty}\left[\partial_{c} f_{s} \frac{d s}{d x} \delta c\left(\mathbf{x}_{s}\right)+f_{s}(c) \delta \frac{d s}{d x}\right] d x \\
& =\int_{-\infty}^{\infty}\left[\partial_{c} f_{s}\left(\nabla c \cdot \delta \mathbf{x}_{s}+\eta\right) \frac{d s}{d x}+f_{s}(c) \partial_{x} h \mathbf{t} \cdot \nabla \zeta\right] d x,
\end{aligned}
$$

where we used Eq. (A9) and applied 


$$
\frac{\partial_{x} \zeta(x)}{(d s / d x)}=\frac{d \zeta(x)}{d x} \frac{d x}{d s}=\frac{d \zeta(x)}{d s}=\mathbf{t} \cdot \nabla \zeta .
$$

The last step is correct if the fields that are only defined at the surface (here $\zeta$, but valid also for $h, \mathbf{n}, \mathbf{t}$, etc.) are interpreted as being defined everywhere with values independent of $z$.

Next, integration by parts has to be applied to eliminate derivatives of the variations. One uses

$$
\begin{aligned}
\int a(\mathbf{t} \cdot \nabla b) d s & =\int a(\mathbf{t} \cdot \nabla b) \frac{d s}{d x} d x \\
& =-\int b \nabla \cdot\left[\mathbf{t} \frac{d s}{d x} a\right] d x \\
& =-\int b(\mathbf{t} \cdot \nabla a) d s .
\end{aligned}
$$

Assuming laterally periodic or localized structures, surface terms resulting from the integration by parts are zero here. We obtain

$$
\begin{aligned}
\delta F_{s}^{\text {top }}= & \int_{-\infty}^{\infty}\left\{\partial_{c} f_{s}\left(\nabla c \cdot \delta \mathbf{x}_{s}+\eta\right)\right. \\
& \left.-\mathbf{t} \cdot \nabla\left[f_{s}(c) \frac{d x}{d s} \partial_{x} h\right] \zeta\right\} d s .
\end{aligned}
$$

Performing derivatives and substituting from Eq. (A10) we arrive at

$$
\begin{aligned}
\delta F_{s}^{\mathrm{top}}= & \int_{-\infty}^{\infty}\left\{\partial_{c} f_{s}\left[\left(\partial_{z} c\right) \zeta+\eta\right]-\left[\left(\mathbf{t} \cdot \nabla f_{s}\right) \partial_{x} h\right.\right. \\
& \left.\left.-f_{s} \kappa\right] \frac{d x}{d s} \zeta\right\} d s,
\end{aligned}
$$

where

$$
\kappa=-\frac{\partial_{x x} h}{\left[1+\left(\partial_{x} h\right)^{2}\right]^{3 / 2}}
$$

is the curvature. Note that $\kappa$ is positive for a convex surface of the fluid. Terms with $\left(\partial_{c} f_{s}\right) \zeta$ can be simplified as follows:

$$
\left(\partial_{c} f_{s}\right)\left[\left(\partial_{z} c\right) \frac{d s}{d x}-(\mathbf{t} \cdot \nabla c) \partial_{x} h\right] \frac{d x}{d s} \zeta=\left(\partial_{c} f_{s}\right)(\mathbf{n} \cdot \nabla c) \frac{d x}{d s} \zeta
$$

resulting in the final expression

$$
\delta F_{s}^{\text {top }}=\int_{-\infty}^{\infty}\left\{\left[(\mathbf{n} \cdot \nabla c) \partial_{c} f_{s}+f_{s} \kappa\right] \frac{d x}{d s} \zeta+\left(\partial_{c} f_{s}\right) \eta\right\} d s .
$$

The variation of the contribution of the free energy at the bottom part of the boundary $\partial \Omega$, denoted by $\delta F_{s}^{\text {bot }}$, can be obtained as a special case of $\delta F_{s}^{\text {top }}$. In Eq. (A11) we consider $\mathbf{x}_{s}(x)=(x, 0), \delta \mathbf{x}_{s}=0, d s / d x=1$ resulting in

$$
\delta F_{s}^{\mathrm{bot}}=\int_{-\infty}^{\infty} \partial_{c} f_{s} \eta d x .
$$

Next, writing $\delta F=\delta F_{b}+\delta F_{s}^{\mathrm{top}}+\delta F_{s}^{\mathrm{bot}}=0$, one is in principle ready to extract governing equations and natural boundary conditions of the problem. Inspecting the form of Eqs. (A7), (A18), and (A19) one notes that we obtained two scalar boundary conditions on the free surface as the prefactors of arbitrary admissible functions $\eta, \zeta$ in the boundary integral of the stationarity condition $\delta F=0$,

$$
\begin{aligned}
& \sigma_{c} \mathbf{n} \cdot \nabla c+\partial_{c} f_{s}=0, \\
& \frac{\sigma_{c}}{2}(\nabla c)^{2}+f(c)-\lambda_{d} c_{1}-\lambda+(\mathbf{n} \cdot \nabla c) \partial_{c} f_{s}+f_{s} \kappa=0,
\end{aligned}
$$

respectively. In order to obtain force boundary conditions, we need to express our variations $\zeta, \eta$ in terms of the virtual displacements because mechanical forces are energetically conjugated to them.

For this purpose, we introduce the variation of the position of a fluid element due to convective motion. The varied Euler coordinates $\mathbf{x}$ of a fluid element specified by its material (Lagrange) coordinates $\mathbf{X}$ can be expressed as

$$
\begin{aligned}
& \mathbf{x}(\mathbf{X} ; \alpha)=\mathbf{X}+\alpha \boldsymbol{\chi}(\mathbf{X}), \\
& \delta \mathbf{x}(\mathbf{X})=\boldsymbol{\chi}(\mathbf{X}),
\end{aligned}
$$

where $\boldsymbol{\chi}=\left(\chi_{x}, \chi_{z}\right)$ is an arbitrary admissible displacement vector. Both coordinate systems coincide for $\alpha=0$. The free surface has to follow the fluid as is expressed by the kinematic condition Eq. (77). This introduces a dependency between the variations $\zeta$ and $\boldsymbol{\chi}$, i.e.,

$$
\begin{aligned}
& \delta h(x)=\zeta(x)=-\partial_{x} h(x) \chi_{x}(x)+\chi_{z}(x), \quad \text { or } \\
& \zeta \mathbf{e}_{z} \cdot \mathbf{n}=\zeta \frac{d x}{d s}=\boldsymbol{\chi} \cdot \mathbf{n} .
\end{aligned}
$$

Next, we consider a fluid element identified by its referential position $\mathbf{X}$. The varied concentration at this element can be expressed using Eq. (A22) as

$$
c(\mathbf{x} ; \alpha)=c[\mathbf{X}+\alpha \boldsymbol{\chi}(\mathbf{X})]+\alpha \eta[\mathbf{X}+\alpha \boldsymbol{\chi}(\mathbf{X})],
$$

being consistent with Eq. (A5). Consider for a moment that no diffusion is active. In that case the concentration $c(\mathbf{x} ; \alpha)$ changes only due to convection described by $\boldsymbol{\chi}$. As a consequence, the concentration at the arbitrary but fixed fluid element $\mathbf{X}$ should remain constant, i.e.,

$$
\begin{aligned}
& \frac{d}{d \alpha}\{c[\mathbf{X}+\alpha \boldsymbol{\chi}(\mathbf{X})]+\alpha \eta[\mathbf{X}+\alpha \boldsymbol{\chi}(\mathbf{X})]\} \\
& \quad=\nabla c[\mathbf{X}+\alpha \boldsymbol{\chi}(\mathbf{X})] \cdot \boldsymbol{\chi}(\mathbf{X})+\eta[\mathbf{X}+\alpha \boldsymbol{\chi}(\mathbf{X})]=0 .
\end{aligned}
$$

We denote the variation $\eta$ that satisfies this condition as $\eta_{\text {co }}$ (convective) and the remaining part as $\eta_{\mathrm{di}}$ (diffusive) variation. As a consequence, we have

$$
\eta=\eta_{\mathrm{co}}+\eta_{\mathrm{di}}=-\nabla c \cdot \boldsymbol{\chi}+\eta_{\mathrm{di}},
$$

where $\eta_{\mathrm{di}}$ is a variation independent of $\boldsymbol{\chi}$ because it is caused by a different physical process. 
Using Eqs. (A27) and (A24), we write the stationarity conditions for the variation $\delta F=\delta F_{b}+\delta F_{s}$. Prefactors of the variations in the bulk and at the free and bottom surface give the Euler-Lagrange equations of the problem. The prefactor of $\eta_{\mathrm{di}}$ in the bulk integral gives

$$
-\sigma_{c} \Delta c+\partial_{c} f-\frac{\lambda_{d}}{2}=0
$$

i.e., the correct static limit of the $2 \mathrm{D}$ version of Eq. (60). From this equation we deduce that $-2 \sigma_{c} \Delta c+2 \partial_{c} f=-\sigma_{c_{1}} \Delta c_{1}$ $+\tilde{\mu}_{d}=\lambda_{d}$ is the chemical potential for a heterogeneous equilibrium discussed in Sec. II C. The prefactor of $\boldsymbol{\chi}$ gives Eq. (A28) multiplied by $\nabla c$. It is the static limit of the bulk momentum equation, i.e., the bulk force equilibrium Eq. (61) with $\mathbf{v}=0$. We can see that if we substitute for $\mu_{d} \rho_{1}=\partial_{c} f(c)$ $\times(c+1)$ in Eq. (51) and put the resulting expression for $p$ into Eq. (62), simplify it using Eq. (A28) (which holds with respect to $\delta c$ ), and use the result to eliminate $p_{\text {eff }}$ in Eq. (61). Finally, after performing the divergence operation we arrive at Eq. (A28) multiplied by $\nabla c$.

The surface integrals yield as the prefactor of $\eta_{\mathrm{di}}$,

$\sigma_{c} \mathbf{n} \cdot \nabla c+\partial_{c} f_{s}=0$,

i.e., the static limit of the $2 \mathrm{D}$ version of Eq. (66) with $\sigma^{-}=0$ and of Eq. (69) with $\sigma^{+}=0$. On the free surface the vectorial prefactor of $\boldsymbol{\chi}$,

$$
\begin{gathered}
(\mathbf{n} \cdot \nabla c)\left(\partial_{c} f_{s}\right) \mathbf{n}-(\nabla c) \partial_{c} f_{s}+f_{s} \kappa \mathbf{n}-\sigma_{c} \mathbf{n} \cdot(\nabla c)(\nabla c) \\
+\left[\frac{\sigma_{c}}{2}(\nabla c)^{2}+f(c)-\lambda_{d} c_{1}-\lambda\right] \mathbf{n}=0
\end{gathered}
$$

gives, using $\underline{\mathbf{I}}-\mathbf{n n}=\mathbf{t t}$ and reordering,

$$
-\sigma_{c} \mathbf{n} \cdot(\nabla c)(\nabla c)-p_{\mathrm{eff}} \mathbf{n}=\mathbf{t t} \cdot \nabla f_{s}-f_{s} \kappa \mathbf{n}
$$

with

$$
p_{\text {eff }}=\left(\partial_{c} f-\sigma_{c} \Delta c\right)(c+1)-\frac{\sigma_{c}}{2}(\nabla c)^{2}-f(c)+\lambda,
$$

where we used Eq. (A28) for $\lambda_{d}$. We proceed to identify the quantities in $p_{\text {eff. }}$. Using Eq. (51), we substitute the free energy density $f(c)$ in Eq. (A32) and simplify to

$$
p_{\text {eff }}=p-\mu_{2} \rho-\sigma_{c}(c+1) \Delta c-\frac{\sigma_{c}}{2}(\nabla c)^{2}+\lambda,
$$

taking into account that $2\left(\partial_{c} f\right) c_{1}=\tilde{\mu}_{d} c_{1}=\mu_{d} \rho_{1}$. The pressure $p_{\text {eff }}$ in Eq. (A33) coincides with the one defined in Eq. (62) in case that

$$
\lambda=\mu_{2} \rho .
$$

We conclude that Eq. (A31) gives the tangential and the normal force equilibrium conditions at the free surface and corresponds to the static limit of the boundary conditions (71) with Eq. (63).

${ }^{1}$ P.-G. de Gennes, "Wetting: Statistics and dynamics," Rev. Mod. Phys. 57, 827 (1985)

${ }^{2}$ G. F. Teletzke, H. T. Davis, and L. E. Scriven, "Wetting hydrodynamics," Rev. Phys. Appl. 23, 989 (1988).

${ }^{3}$ A. Oron, S. H. Davis, and S. G. Bankoff, "Long-scale evolution of thin liquid films," Rev. Mod. Phys. 69, 931 (1997).
${ }^{4}$ M. Geoghegan and G. Krausch, "Wetting at polymer surfaces and interfaces," Prog. Polym. Sci. 28, 261 (2003).

${ }^{5} \mathrm{P}$. Müller-Buschbaum, "Dewetting and pattern formation in thin polymer films as investigated in real and reciprocal space," J. Phys.: Condens. Matter 15, R1549 (2003).

${ }^{6} \mathrm{U}$. Thiele, "Open questions and promising new fields in dewetting," Eur. Phys. J. E 12, 409 (2003).

${ }^{7}$ S. J. Weinstein and K. J. Ruschak, "Coating flows," Annu. Rev. Fluid Mech. 36, 29 (2004).

${ }^{8}$ R. Seemann, S. Herminghaus, C. Neto, S. Schlagowski, D. Podzimek, R. Konrad, H. Mantz, and K. Jacobs, "Dynamics and structure formation in thin polymer melt films," J. Phys.: Condens. Matter 17, S267 (2005).

${ }^{9}$ Thin Films of Soft Matter, edited by S. Kalliadasis and U. Thiele (Springer, Wien, 2007), ISBN 978-3-211-69807-5, CISM 490.

${ }^{10}$ H. A. Stone, A. D. Stroock, and A. Ajdari, "Engineering flows in small devices: Microfluidics toward a lab-on-a-chip," Annu. Rev. Fluid Mech. 36, 381 (2004).

${ }^{11}$ T. M. Squires and S. R. Quake, "Microfluidics: Fluid physics at the nanoliter scale," Rev. Mod. Phys. 77, 977 (2005).

${ }^{12}$ G. Reiter, "Dewetting of thin polymer films," Phys. Rev. Lett. 68, 75 (1992).

${ }^{13}$ A. Sharma and G. Reiter, "Instability of thin polymer films on coated substrates: Rupture, dewetting and drop formation," J. Colloid Interface Sci. 178, 383 (1996).

${ }^{14}$ J. Becker, G. Grün, R. Seemann, H. Mantz, K. Jacobs, K. R. Mecke, and R. Blossey, "Complex dewetting scenarios captured by thin-film models," Nat. Mater. 2, 59 (2003).

${ }^{15}$ E. Ruckenstein and R. K. Jain, "Spontaneous rupture of thin liquid films," J. Chem. Soc., Faraday Trans. 2 70, 132 (1974).

${ }^{16}$ V. S. Mitlin, "Dewetting of solid surface: Analogy with spinodal decomposition," J. Colloid Interface Sci. 156, 491 (1993).

${ }^{17}$ U. Thiele, M. G. Velarde, and K. Neuffer, "Dewetting: Film rupture by nucleation in the spinodal regime," Phys. Rev. Lett. 87, 016104 (2001).

${ }^{18} \mathrm{M}$. Bestehorn and K. Neuffer, "Surface patterns of laterally extended thin liquid films in three dimensions," Phys. Rev. Lett. 87, 046101 (2001).

${ }^{19}$ K. Kargupta, R. Konnur, and A. Sharma, "Spontaneous dewetting and ordered patterns in evaporating thin liquid films on homogeneous and heterogeneous substrates," Langmuir 17, 1294 (2001).

${ }^{20}$ F. Saulnier, E. Raphaël, and P.-G. de Gennes, "Dewetting of thin-film polymers," Phys. Rev. E 66, 061607 (2002).

${ }^{21}$ T. Kerle, J. Klein, and R. Yerushalmi-Rozen, "Accelerated rupture at the liquid/liquid interface," Langmuir 18, 10146 (2002).

${ }^{22} \mathrm{R}$. Yerushalmi-Rozen, T. Kerle, and J. Klein, "Alternative dewetting pathways of thin liquid films," Science 285, 1254 (1999).

${ }^{23}$ M. Oron, T. Kerle, R. Yerushalmi-Rozen, and J. Klein, "Persistent droplet motion in liquid-liquid dewetting," Phys. Rev. Lett. 92, 236104 (2004).

${ }^{24}$ R. D. Deegan, O. Bakajin, T. F. Dupont, G. Huber, S. R. Nagel, and T. A. Witten, "Capillary flow as the cause of ring stains from dried liquid drops," Nature (London) 389, 827 (1997).

${ }^{25}$ M. Mertig, U. Thiele, J. Bradt, D. Klemm, and W. Pompe, "Dewetting of thin collagenous precursor films," Appl. Phys. A A66, S565 (1998).

${ }^{26} \mathrm{U}$. Thiele, M. Mertig, and W. Pompe, "Dewetting of an evaporating thin liquid film: Heterogeneous nucleation and surface instability," Phys. Rev. Lett. 80, 2869 (1998).

${ }^{27}$ O. Pitois and B. Francois, "Crystallization of condensation droplets on a liquid surface," Colloid Polym. Sci. 277, 574 (1999).

${ }^{28}$ L. Weh, "Self-organized structures at the surface of thin polymer films," Mater. Sci. Eng., C 8-9, 463 (1999).

${ }^{29}$ R. D. Deegan, "Pattern formation in drying drops," Phys. Rev. E 61, 475 (2000).

${ }^{30}$ M. Yamamura, T. Nishio, T. Kajiwara, and K. Adachi, "Evaporationinduced pattern formation in polymer films via secondary phase separation," Chem. Eng. Sci. 57, 2901 (2002).

${ }^{31}$ X. Gu, D. Raghavan, J. F. Douglas, and A. Karim, "Hole-growth instability in the dewetting of evaporating polymer solution films," J. Polym. Sci., Part B: Polym. Phys. 40, 2825 (2002).

${ }^{32}$ P. Moriarty, M. D. R. Taylor, and M. Brust, "Nanostructured cellular networks," Phys. Rev. Lett. 89, 248303 (2002).

${ }^{33}$ E. Rabani, D. R. Reichman, P. L. Geissler, and L. E. Brus, "Dryingmediated self-assembly of nanoparticles," Nature (London) 426, 271 (2003).

${ }^{34}$ C. P. Martin, M. O. Blunt, E. Pauliac-Vaujour, A. Stannard, P. Moriarty, I. Vanceat, and U. Thiele, "Controlling pattern formation in nanoparticle assemblies via directed solvent dewetting," Phys. Rev. Lett. 99, 116103 
(2007).

${ }^{35}$ E. Bormashenko, R. Pogreb, O. Stanevsky, Y. Bormashenko, T. Stein, and O. Gengelman, "Mesoscopic patterning in evaporated polymer solutions: New experimental data and physical mechanisms," Langmuir 21, 9604 (2005).

${ }^{36}$ E. Bormashenko, R. Pogreb, O. Stanevsky, Y. Bormashenko, S. Tamir, R. Cohen, M. Nunberg, V. Z. Gaisin, M. Gorelik, and O. V. Gendelman, "Mesoscopic and submicroscopic patterning in thin polymer films: Impact of the solvent," Mater. Lett. 59, 2461 (2005).

${ }^{37}$ L. V. Govor, J. Parisi, G. H. Bauer, and G. Reiter, "Instability and droplet formation in evaporating thin films of a binary solution," Phys. Rev. E 71, 051603 (2005)

${ }^{38}$ S. Y. Heriot and R. A. L. Jones, "An interfacial instability in a transient wetting layer leads to lateral phase separation in thin spin-cast polymerblend films," Nat. Mater. 4, 782 (2005).

${ }^{39}$ P. Müller-Buschbaum, E. Bauer, S. Pfister, S. V. Roth, M. Burghammer, C. Riekel, C. David, and U. Thiele, "Creation of multiscale stripe-like patterns in thin polymer blend films," Europhys. Lett. 73, 35 (2006).

${ }^{40}$ A. Pototsky, M. Bestehorn, D. Merkt, and U. Thiele, "Alternative pathways of dewetting for a thin liquid two-layer film," Phys. Rev. E 70, 025201(R) (2004).

${ }^{41}$ L. S. Fisher and A. A. Golovin, "Nonlinear stability analysis of a twolayer thin liquid film: dewetting and autophobic behavior," J. Colloid Interface Sci. 291, 515 (2005).

${ }^{42}$ A. Pototsky, M. Bestehorn, D. Merkt, and U. Thiele, "Morphology changes in the evolution of liquid two-layer films," J. Chem. Phys. 122, 224711 (2005).

${ }^{43}$ D. Bandyopadhyay, R. Gulabani, and A. Sharma, "Stability and dynamics of bilayers," Ind. Eng. Chem. Res. 44, 1259 (2005).

${ }^{44}$ A. Pototsky, M. Bestehorn, D. Merkt, and U. Thiele, "3D surface patterns in liquid two-layer films," Europhys. Lett. 74, 665 (2006).

${ }^{45}$ D. Merkt, A. Pototsky, M. Bestehorn, and U. Thiele, "Long-wave theory of bounded two-layer films with a free liquid-liquid interface: Short- and long-time evolution,” Phys. Fluids 17, 064104 (2005).

${ }^{46} \mathrm{~K}$. Kargupta and A. Sharma, "Templating of thin films induced by dewetting on patterned surfaces," Phys. Rev. Lett. 86, 4536 (2001).

${ }^{47} \mathrm{~K}$. Kargupta and A. Sharma, "Creation of ordered patterns by dewetting of thin films on homogeneous and heterogeneous substrates," J. Colloid Interface Sci. 245, 99 (2002).

${ }^{48}$ L. Brusch, H. Kühne, U. Thiele, and M. Bär, "Dewetting of thin films on heterogeneous substrates: Pinning vs coarsening," Phys. Rev. E 66, 011602 (2002)

${ }^{49}$ U. Thiele, L. Brusch, M. Bestehorn, and M. Bär, "Modelling thin-film dewetting on structured substrates and templates: Bifurcation analysis and numerical simulations," Eur. Phys. J. E 11, 255 (2003).

${ }^{50} \mathrm{U}$. Thiele and E. Knobloch, "On the depinning of a driven drop on a heterogeneous substrate," New J. Phys. 8, 313 (2006).

${ }^{51}$ M. R. E. Warner, R. V. Craster, and O. K. Matar, "Surface patterning via evaporation of ultrathin films containing nanoparticles," J. Colloid Interface Sci. 267, 92 (2003).

${ }^{52}$ J. P. Burelbach, S. G. Bankoff, and S. H. Davis, "Nonlinear stability of evaporating/condensing liquid films," J. Fluid Mech. 195, 463 (1988).

${ }^{53}$ N. Samid-Merzel, S. G. Lipson, and D. S. Tannhauser, "Pattern formation in drying water films," Phys. Rev. E 57, 2906 (1998).

${ }^{54}$ K. D. Danov, V. N. Paunov, N. Alleborn, H. Raszillier, and F. Durst, "Stability of evaporating two-layered liquid film in the presence of surfactant. I. The equations of lubrication approximation," Chem. Eng. Sci. 53, 2809 (1998).

${ }^{55}$ A. Oron and S. G. Bankoff, "Dewetting of a heated surface by an evaporating liquid film under conjoining/disjoining pressures," J. Colloid Interface Sci. 218, 152 (1999).

${ }^{56}$ L. M. Pismen, "Spinodal dewetting in a volatile liquid film," Phys. Rev. E 70, 021601 (2004).

${ }^{57}$ F. Bruder and R. Brenn, "Spinodal decomposition in thin-films of a polymer blend," Phys. Rev. Lett. 69, 624 (1992).

${ }^{58}$ G. Krausch, C. A. Dai, E. J. Kramer, J. F. Marko, and F. S. Bates, "Interference of spinodal waves in thin polymer films," Macromolecules 26, 5566 (1993).

${ }^{59}$ U. Steiner, J. Klein, and L. J. Fetters, "Surface phase inversion in finitesized binary-mixtures," Phys. Rev. Lett. 72, 1498 (1994).

${ }^{60}$ C. Huang, M. Olvera de la Cruz, and B. W. Swift, "Phase-separation of ternary mixtures: Symmetrical polymer blends," Macromolecules 28, 7996 (1995).

${ }^{61}$ M. Sprenger, S. Walheim, A. Budkowski, and U. Steiner, "Hierarchic structure formation in binary and ternary polymer blends," Interface Sci. 11, 225 (2003).

${ }^{62}$ J. Genzer and E. J. Kramer, "Wetting of substrates with phase-separated binary polymer mixtures," Phys. Rev. Lett. 78, 4946 (1997).

${ }^{63}$ K. D. Jandt, J. Heier, F. S. Bates, and E. J. Kramer, "Transient surface roughening of thin films of phase separating polymer mixtures," Langmuir 12, 3716 (1996).

${ }^{64}$ A. Karim, J. F. Douglas, B. P. Lee, S. C. Glotzer, J. A. Rogers, R. J. Jackman, E. J. Amis, and G. M. Whitesides, "Phase separation of ultrathin polymer-blend films on patterned substrates," Phys. Rev. E 57, R6273 (1998).

${ }^{65} \mathrm{U}$. Steiner and J. Klein, "Growth of wetting layers from liquid mixtures," Phys. Rev. Lett. 77, 2526 (1996).

${ }^{66} \mathrm{H}$. Wang and R. J. Composto, "Understanding morphology evolution and roughening in phase-separating thin-film polymer blends," Europhys. Lett. 50, $622(2000)$.

${ }^{67} \mathrm{H}$. Wang and R. J. Composto, "Wetting and phase separation in polymer blend films: Identification of four thickness regimes with distinct morphological pathways," Interface Sci. 11, 237 (2003).

${ }^{68}$ R. A. L. Jones, L. J. Norton, E. J. Kramer, F. S. Bates, and P. Wiltzius, "Surface-directed spinodal decomposition," Phys. Rev. Lett. 66, 1326 (1991).

${ }^{69}$ J. Genzer and E. J. Kramer, "Pretransitional thinning of a polymer wetting layer," Europhys. Lett. 44, 180 (1998).

${ }^{70} \mathrm{H}$. Wang and R. J. Composto, "Thin film polymer blends undergoing phase separation and wetting: Identification of early, intermediate, and late stages," J. Chem. Phys. 113, 10386 (2000).

${ }^{71}$ G. Nisato, B. D. Ermi, J. F. Douglas, and A. Karim, "Excitation of surface deformation modes of a phase-separating polymer blend on a patterned substrate," Macromolecules 32, 2356 (1999).

${ }^{72}$ J. W. Cahn and J. E. Hilliard, "Free energy of a nonuniform system. 1. Interfacual free energy," J. Chem. Phys. 28, 258 (1958).

${ }^{73}$ H. P. Fischer, P. Maass, and W. Dieterich, "Novel surface modes in spinodal decomposition," Phys. Rev. Lett. 79, 893 (1997).

${ }^{74}$ H. P. Fischer, P. Maass, and W. Dieterich, "Diverging time and length scales of spinodal decomposition modes in thin films," Europhys. Lett. 42, 49 (1998).

${ }^{75}$ R. Kenzler, F. Eurich, P. Maass, B. Rinn, J. Schropp, E. Bohl, and W. Dieterich, "Phase separation in confined geometries: Solving the CahnHilliard equation with generic boundary conditions," Comput. Phys. Commun. 133, 139 (2001).

${ }^{76}$ P. C. Hohenberg and B. I. Halperin, "Theory of dynamic critical phenomena," Rev. Mod. Phys. 49, 435 (1977).

${ }^{77}$ D. M. Anderson, G. B. McFadden, and A. A. Wheeler, "Diffuse-interface methods in fluid mechanics," Annu. Rev. Fluid Mech. 30, 139 (1998).

${ }^{78}$ D. Jasnow and J. Viñals, "Coarse-grained description of thermocapillary flow," Phys. Fluids 8, 660 (1996).

${ }^{79} \mathrm{~N}$. Vladimirova, A. Malagoli, and R. Mauri, "Two-dimensional model of phase segregation in liquid binary mixtures," Phys. Rev. E 60, 6968 (1999).

${ }^{80} \mathrm{~N}$. Vladimirova, A. Malagoli, and R. Mauri, "Diffusiophoresis of twodimensional liquid droplets in a phase separating system," Phys. Rev. E 60, 2037 (1999).

${ }^{81}$ J. Lowengrub and L. Truskinovsky, "Quasi-incompressible Cahn-Hilliard fluids and topological transitions," Proc. R. Soc. London, Ser. A 454, 2617 (1998).

${ }^{82}$ D. J. Korteweg, "Sur la forme que prennent les équations dumouvement des fluides si l'on tient compte des forces capillaires causées par des variations de densité," Arch. Neerl. Sci. Exactes Nat., Ser. II 6, 1 (1901).

${ }^{83}$ D. D. Joseph, "Fluid-dynamics of 2 miscible liquids with diffusion and gradient stresses," Eur. J. Mech. B/Fluids 9, 565 (1990).

${ }^{84}$ L. M. Pismen and Y. Pomeau, "Disjoining potential and spreading of thin liquid layers in the diffuse interface model coupled to hydrodynamics," Phys. Rev. E 62, 2480 (2000).

${ }^{85}$ L. M. Pismen, "Nonlocal diffuse interface theory of thin films and the moving contact line," Phys. Rev. E 64, 021603 (2001).

${ }^{86} \mathrm{C}$. Rohde, "On local and non-local Navier-Stokes-Korteweg systems for liquid-vapour phase transitions," Z. Angew. Math. Mech. 85, 839 (2005).

${ }^{87}$ L. K. Antanovskii, "Microscale theory of surface tension," Phys. Rev. E 54, 6285 (1996).

${ }^{88}$ R. Borcia and M. Bestehorn, "Phase-field model for Marangoni convection in liquid-gas systems with a deformable interface," Phys. Rev. E 67, 066307 (2003). 
${ }^{89}$ L. K. Antanovskii, "A phase field model of capillarity," Phys. Fluids 7, 747 (1995).

${ }^{90} \mathrm{H}$. Tanaka, "Interplay between wetting and phase separation in binary fluid mixtures: Roles of hydrodynamics," J. Phys.: Condens. Matter 13, 4637 (2001).

${ }^{91}$ G. P. Galdi, D. D. Joseph, L. Preziosi, and S. Rionero, "Mathematical problems for miscible, incompressible, fluids with Korteweg stresses," Eur. J. Mech. B/Fluids 10, 253 (1991).

${ }^{92}$ O. Kuksenok, D. Jasnow, and A. C. Balazs, "Diffusive intertwining of two fluid phases in chemically patterned microchannels," Phys. Rev. E 68, 051505 (2003).

${ }^{93} \mathrm{M}$. De Menech, "Modeling of droplet breakup in a microfluidic T-shaped junction with a phase-field model," Phys. Rev. E 73, 031505 (2006).

${ }^{94}$ H. P. Fischer and W. Dieterich, "Early-time kinetics of ordering in the presence of interactions with a concentration field," Phys. Rev. E 56, 6909 (1997).

${ }^{95}$ K. Binder, "Spinodal decomposition in confined geometry," J. NonEquilib. Thermodyn. 23, 1 (1998).

${ }^{96}$ H. L. Frisch, P. Nielaba, and K. Binder, "Surface effects on spinodal decomposition in the framework of a linearized theory," Phys. Rev. E 52, 2848 (1995).

${ }^{97}$ N. Clarke, "Instabilities in thin-film binary mixtures," Eur. Phys. J. E 14, 207 (2004)

${ }^{98} \mathrm{~N}$. Clarke, "Toward a model for pattern formation in ultrathin-film binary mixtures," Macromolecules 38, 6775 (2005).

${ }^{99}$ M. Plapp and J. F. Gouyet, "Surface modes and ordered patterns during spinodal decomposition of an abv model alloy," Phys. Rev. Lett. 78, 4970 (1997).

${ }^{100}$ M. Plapp and J. F. Gouyet, "Spinodal decomposition of an abv model alloy: Patterns at unstable surfaces,” Eur. Phys. J. B 9, 267 (1999).

${ }^{101}$ H. Furukawa, "Dynamics of phase separation of a simple fluid mixture: Comparison between molecular dynamics and numerical integration of the phenomenological equation," Phys. Rev. E 55, 1150 (1997)

${ }^{102}$ F. R. S. Batchelor, An Introduction to Fluid Dynamics (Cambridge University Press, Cambridge, 2000).

${ }^{103}$ L. D. Landau and E. M. Lifshitz, Hydrodynamics, 2nd ed. (Pergamon, Oxford, 1987).

${ }^{104}$ H. Ding, P. D. M. Spelt, and C. Shu, "Diffuse interface model for incompressible two-phase flows with large density ratios," J. Comput. Phys. 226, 2078 (2007).

${ }^{105}$ D. A. Edwards, H. Brenner, and D. T. Wasan, Interfacial Transport Processes and Rheology (Butterworth-Heinemann, Stoneheam, 1991).

${ }^{106}$ A. A. Golovin, A. A. Nepomnyashchy, S. H. Davis, and M. A. Zaks, "Convective Cahn-Hilliard models: from coarsening to roughening," Phys. Rev. Lett. 86, 1550 (2001).

${ }^{107}$ S. J. Watson, F. Otto, B. Y. Rubinstein, and S. H. Davis, "Coarsening dynamics of the convective Cahn-Hilliard equation," Physica D 178, 127 (2003).

${ }^{108}$ A. G. Lamorgese and R. Mauri, "Mixing of macroscopically quiescent liquid mixtures," Phys. Fluids 18, 044107 (2006).

${ }^{109}$ D. Molin and R. Mauri, "Enhanced heat transport during phase separation of liquid binary mixtures," Phys. Fluids 19, 074102 (2007).

${ }^{110}$ A. Oron and P. Rosenau, "Formation of patterns induced by thermocapillarity and gravity,” J. Phys. II 2, 131 (1992).

${ }^{111}$ A. Oron and P. Rosenau, "On a nonlinear thermocapillary effect in thin liquid layers," J. Fluid Mech. 273, 361 (1994).

${ }^{112}$ A. Novick-Cohen and L. A. Segel, "Nonlinear aspects of the CahnHilliard equation," Physica D 10, 277 (1984).

${ }^{113}$ U. Thiele, M. G. Velarde, K. Neuffer, and Y. Pomeau, "Film rupture in the diffuse interface model coupled to hydrodynamics," Phys. Rev. E 64, 031602 (2001).

${ }^{114}$ E. J. Doedel, A. R. Champneys, T. F. Fairgrieve, Y. A. Kuznetsov, B. Sandstede, and X. J. Wang, AUTO97: Continuation and Bifurcation Software for Ordinary Differential Equations (Concordia University, Montreal, 1997). 